\title{
EXISTENCE OF MARTINGALE AND STATIONARY SUITABLE WEAK SOLUTIONS FOR A STOCHASTIC NAVIER-STOKES SYSTEM
}

\author{
MARCO ROMITO
}

\begin{abstract}
The existence of suitable weak solutions of 3D NavierStokes equations, driven by a random body force, is proved. These solutions satisfy a local balance of energy. Moreover it is proved also the existence of a statistically stationary solution.
\end{abstract}

\section{INTRODUCTION}

The suitable weak solutions of three dimensional Navier-Stokes system are weak solutions which satisfy a local energy inequality. The local energy inequality can be seen as a mathematical counterpart of a local balance of the variation of the energy of the fluid governed by the Navier-Stokes equations. This additional property in general is not verified by solutions to Navier-Stokes equations (e.g. solutions obtained by Galerkin approximations), but, from a physical viewpoint, it is natural to choose solutions which have more regularity properties and so a more precise physical meaning.

The concept of suitable weak solution was introduced firstly by Caffarelli, Kohn and Nirenberg in [4], with the aim to study the partial regularity of solutions of Navier-Stokes system. With this approach the local energy inequality is turned out to be a crucial tool and it has brought Caffarelli, Kohn and Nirenberg to show in [4] the best known result in this setting.

The suitable weak solutions were already defined in the papers of Scheffer [16], [17], [18], even if in a rather implicit way. Scheffer gives an interpretation of the local energy inequality in terms of the existence of an external force $f$ acting against the flow, in other words

$$
f(t, x) \cdot u(t, x) \leq 0 .
$$

In this paper we will show the existence of martingale suitable weak solutions of three dimensional Navier-Stokes system. These are solutions of the Navier-Stokes equations driven by a random body force (a white noise). Different interpretations can be given to these terms. A random force can represent all those phenomena that are usually neglected where the system

Date: January 11, 2001.

1991 Mathematics Subject Classification. Primary 76D05; Secondary 35R60.

Key words and phrases. Navier-Stokes equations, suitable weak solutions martingale solutions, stationary solutions. 
is stable. A random solution of the Navier-Stokes equations can take into account that flows with a large Reynolds number are chaotic and sensitive to microscopic perturbations. Sources of perturbations can be the roughness of the boundary, or the effects of the environment external to the system, such as acoustic waves, etc.

We will prove also the existence of stationary suitable weak solutions, where stationary has to be understood in a statistical sense. These solutions may describe a fluid in a turbulent regime. The partial regularity of these solutions will be studied in other papers (see [7], [8] and [15]).

Many authors have proved the existence of deterministic suitable weak solutions, see for example Caffarelli, Kohn and Nirenberg [4], or P.L. Lions [13], Beirao da Veiga [2], Lemarie-Rieusset [11]. None of the methods used in these papers is well suited to prove the existence of martingale solutions. The existence of suitable weak solutions in a stochastic setting seems to be new.

1.1. Notations. Let $D$ be an open bounded subset of $\mathbf{R}^{3}$ having a smooth boundary and for each $T>0$ set $D_{T}=(0, T) \times D$. Define the space

$$
H=\left\{u: D \rightarrow \mathbf{R}^{3}\left|u \in\left(L^{2}(D)\right)^{3}, \operatorname{div} u=0, u \cdot n\right|_{\partial D}=0\right\},
$$

where $n$ is the outer normal to $\partial D$ (see for example Temam [22]), and the space

$$
V=\left\{u \in\left(H^{1}(D)\right)^{3}|\operatorname{div} u=0, u|_{\partial D}=0\right\} .
$$

The $L^{2}$-norm of elements of $H$ will be denoted by $|\cdot|$ and the $H^{1}$-norm of elements of $V$ will be denoted by $\|\cdot\|$. If the space $H$ is identified with its dual and the space $H^{\prime}$ is identified with a subspace of $V^{\prime}$, then

$$
V \subset H \subset V^{\prime} \text {. }
$$

The operator $A: D(A) \subset H \rightarrow H$ is defined as $A u=-\mathcal{P} \triangle u$, where $\mathcal{P}$ is the orthogonal projection from $L^{2}(D)^{3}$ onto $H$ and $D(A)=H^{2}(D)^{3} \cap V$. The operator $A$ is positive self adjoint with compact resolvent. The eigenvalues of $A$ are denoted by $0<\lambda_{1} \leq \lambda_{2} \leq \ldots$ and $e_{1}, e_{2}, \ldots$ is a corresponding complete orthonormal system of eigenvectors.

Moreover, if the dual space of $D(A)$ is denoted by $D\left(A^{-1}\right)$, then identifications as above give the dense continuous inclusions

$$
D(A) \subset V \subset H \subset V^{\prime} \subset D\left(A^{-1}\right) .
$$

The fractional powers $A^{\alpha}$ of $A, \alpha \geq 0$, are simply defined by

$$
A^{\alpha} x=\sum_{i=1}^{\infty} \lambda_{i}^{\alpha}\left\langle x, e_{i}\right\rangle e_{i}
$$

with domain

$$
D\left(A^{\alpha}\right)=\left\{x \in H \mid\|x\|_{D\left(A^{\alpha}\right)}<\infty\right\}
$$


where

$$
\|x\|_{D\left(A^{\alpha}\right)}^{2}=\sum_{i=1}^{\infty} \lambda_{i}^{2 \alpha}\left\langle x, e_{i}\right\rangle^{2}=\left|A^{\alpha} x\right|^{2} .
$$

The space $D\left(A^{\alpha}\right)$ is an Hilbert space with the inner product

$$
\langle x, y\rangle_{D\left(A^{\alpha}\right)}=\left\langle A^{\alpha} x, A^{\alpha} y\right\rangle, \quad x, y \in D\left(A^{\alpha}\right) .
$$

Notice that $D\left(A^{\alpha}\right) \subset H^{2 \alpha}(D)$.

Since $V$ coincides with $D\left(A^{1 / 2}\right)$ (see Temam [23] Section 2.2, or Temam [24], Ch. III, Section 2.1), the space $V$ can be endowed with the norm $\|u\|=\left|A^{1 / 2} u\right|$. The Poincarè inequality gives

$$
\|u\|^{2} \geq \lambda_{1}|u|^{2} \text {. }
$$

Moreover we will consider the Sobolev spaces $W^{s, p}(0, T ; H)$ endowed with the norm

$$
\|f\|_{W^{s, p}}^{p}=\int_{0}^{T}|f|^{p} d t+\int_{0}^{T} \int_{0}^{T} \frac{|f(t)-f(s)|^{p}}{|t-s|^{1+s p}} d s d t
$$

Finally we define the bilinear operator $B: V \times V \rightarrow V^{\prime}$ as

$$
\langle B(u, v), w\rangle=\int_{D} w \cdot(u \cdot \nabla) v d x, \quad w \in V .
$$

The operator $B$ can be extended in many different spaces (see for example Temam [23]).

In the sequel we will largely use the following local Sobolev inequality. Let $u \in H^{1}\left(B_{r}\right)$, then

$$
\int_{B_{r}}|u|^{q} \leq C\left(\int_{B_{r}}|\nabla u|^{2}\right)^{a}\left(\int_{B_{r}}|u|^{2}\right)^{\frac{q}{2}-a}+\frac{C}{r^{2 a}}\left(\int_{B_{r}}|u|^{2}\right)^{\frac{q}{2}},
$$

where $q \in[2,6]$ and $a=\frac{3}{4}(q-2)$.

\section{DEFINITIONS AND MAIN RESULTS}

We consider a viscous incompressible homogeneous Newtonian fluid in the bounded open domain $D \subset \mathbf{R}^{3}$, described by the Navier-Stokes equations

$$
\begin{cases}\partial_{t} u+(u \cdot \nabla) u+\nabla P=\mathrm{v} \Delta u+f+\partial_{t} g & \text { in } D_{T} \\ \operatorname{div} u=0 & \text { in } D_{T} \\ u=0 & \text { on }[0, T] \times \partial D \\ u(0)=u_{0} & \end{cases}
$$

where $u$ is the velocity field, $P$ is the pressure field and $v$ is the kinematic viscosity. For simplicity we will take $v=1$, since its value is not relevant in the present setting. The term $\partial_{t} g$ represents a rapidly fluctuating force and in this paper it will be model by a white noise. In order to handle this term we introduce the new variables

$$
v=u-z, \quad \pi=P-Q,
$$


where the pair $(z, Q)$ solves the following Stokes equation

$$
\left\{\begin{array}{l}
\partial_{t} z+\nabla Q=\triangle z+f+\partial_{t} g, \\
\operatorname{div} z=0, \\
z=0 \\
z(0)=0,
\end{array}\right.
$$

in $[0, T] \times D$. Then the new variables $(v, \pi)$ solve the following equation

$$
\begin{cases}\partial_{t} v+((v+z) \cdot \nabla)(v+z)+\nabla \pi=\Delta v, & \\ \operatorname{div} v=0, & \text { on } \partial D, \\ v=0 & \\ v(0)=u_{0}, & \end{cases}
$$

where the white noise term has disappeared. We will ask for a path-wise local energy inequality to the pair $(v, \pi)$, as we shall see in the sequel.

2.1. Assumptions on the data. We will model the fluctuation part of the body force $\partial_{t} g$ as a noise white in time $\partial_{t} B$, so that $B$ is a Brownian motion. We will assume throughout the paper the following assumptions

$$
u_{0} \in H
$$$$
f \in L^{2}(0, T ; H) \text {, }
$$

$B$ is a Brownian motion with trajectories in $D\left(A^{\delta}\right)$

for a small $\delta>0$. It is possible to see that this set of assumptions implies that the trajectories of the solution $z$ of equations (2.2) have the following regularity properties

$$
z \in L^{\infty}(0, T ; H) \cup L^{2}(0, T ; V) \cap L^{\infty}\left(0, T ; L^{4}(D)\right) \quad \mathbb{P}-\text { a.s. }
$$

(see Flandoli [6]).

Remark 2.1. Another way to give assumptions (As) regarding the Brownian motion $B$ is to consider the covariance operator $O$, which is a positive bounded self-adjoint operator. We suppose that $O$ maps $H$ into $D\left(A^{\delta}\right)$. So a sufficient condition that ensures (AS) is that the operator $A^{\delta} O A^{\delta}$ has a bounded extension to $H$ which is of trace class (see [5] for more).

Remark 2.2. Even if we are mainly interested in interpreting the fluctuation $\partial_{t} g$ as a white-noise, in view of stationary solutions we will consider also deterministic solutions. In this case we will assume

$$
\begin{aligned}
& u_{0} \in H, \\
& f \in L^{2}(0, T ; H), \\
& g \in C^{\frac{1}{2}-\varepsilon}\left([0, T] ; D\left(A^{\delta}\right)\right), \quad g(0)=0,
\end{aligned}
$$

for $\delta>\varepsilon>0$, so that again property (2.4) holds (we refer again to Flandoli [6]). Note that it is possible to choose the function $g$ with different regularity 
properties, combining in different ways the differentiability with respect to time and the differentiability with respect to space.

2.2. Martingale suitable weak solutions. We start with the definition of martingale suitable weak solutions and we give the main theorem about their existence.

Before doing this, we define the suitable solutions in a deterministic setting, so that the derivative of $g$ with respect to time has to be understood in the sense of distributions.

Definition 2.3. Let $T \in(0, \infty]$. A suitable weak solution to Navier-Stokes equations is a pair $(u, P)$ such that if $v=u-z$ and $\pi=P-Q$, where $(z, Q)$ is the solution of equation (2.2), then

1. $v$ is weakly continuous with respect to time,

2. $v \in L^{\infty}(0, T ; H) \cap L^{2}(0, T ; V)$ and $\pi \in L_{\mathrm{loc}}^{5 / 3}\left(D_{T}\right)$,

3. $(v, \pi)$ satisfies equation (2.3) in the sense of distributions on $D_{T}$,

4. for all $t \leq T$ and almost all $s<t$,

$$
|v(t)|^{2}+2 \int_{s}^{t}\|v\|^{2} d r \leq|v(s)|^{2}+\int_{s}^{t} \int_{D} z \cdot((v+z) \cdot \nabla) v d x d r
$$

(v) for any $\phi \in C_{c}^{\infty}\left(D_{T}\right), \phi \geq 0$,

$$
\begin{aligned}
2 \int_{0}^{T} \int_{D}|\nabla v|^{2} \phi \leq & \int_{0}^{T} \int_{D}|v|^{2}\left(\partial_{t} \phi+\triangle \phi\right)+2 \int_{0}^{T} \int_{D} \pi(v \cdot \nabla \phi) \\
& +\int_{0}^{T} \int_{D}\left(|v|^{2}+2 v \cdot z\right)((v+z) \cdot \nabla \phi) \\
& +2 \int_{0}^{T} \int_{D} \phi z \cdot((v+z) \cdot \nabla) v
\end{aligned}
$$

A martingale suitable weak solution for the Navier-Stokes equations will be the solution of a stochastic differential equation driven by an additive noise such that its trajectories are suitable weak solutions in the sense of the definition above. More precisely:

Definition 2.4. A martingale suitable weak solution is a process $(u, P)$ defined on a stochastic basis

$$
\left(\Omega, \mathcal{F},(\mathcal{F})_{t \geq 0}, \mathbb{P},\left(B_{t}\right)_{t \geq 0}\right),
$$

where $B$ is a Brownian motion adapted to the filtration with values in $D\left(A^{\delta}\right)$, such that

$$
\omega \in \Omega \rightarrow(u(\omega), P(\omega)) \in L^{2}(0, T ; H) \times L_{\mathrm{loc}}^{5 / 3}\left(D_{T}\right)
$$

is a measurable mapping and such that there exists a set $\Omega_{0} \subset \Omega$ of full probability such that the pair $(u(\cdot, \omega), P(\cdot, \omega))$ is a suitable weak solution in the sense of Definition 2.3, with respect to the body force $f+\partial_{t} B_{t}(\omega)$, for all $\omega \in \Omega_{0}$. 
We want to explain the meaning of the last part of this definition. Since $B$ is a Brownian motion, under assumption (As) it has $\mathbb{P}$-a.s. trajectories in $C^{\frac{1}{2}-\varepsilon}\left([0, T] ; D\left(A^{\beta}\right)\right)$, for $0<\varepsilon<\beta \leq \delta$. So for every given $\omega \in \Omega_{0}$, we have that $B(\omega) \in C^{\frac{1}{2}-\varepsilon}\left([0, T] ; D\left(A^{\beta}\right)\right)$, the solution $(z(\omega), Q(\omega))$ enjoys the regularity stated in (2.4) and the pair $(u(\omega), P(\omega))$ satisfies all the conditions of Definition 2.3 with respect to these functions.

We can give now the main existence theorem for martingale suitable weak solutions.

Theorem 2.5. Assume (As). There exists a martingale suitable weak solution (in the sense of Definition 2.4 above) with initial data $u_{0}$. Moreover

$$
\mathbb{E}|u(t)|_{H}^{2}+\mathbb{E} \int_{s}^{t}\|u\|_{V}^{2} d r \leq \mathbb{E}|u(s)|_{H}^{2}+\sigma(t-s)+\mathbb{E} \int_{s}^{t}\|f\|_{V^{\prime}} d s,
$$

and

$$
\begin{aligned}
\mathbb{E}\left[\sup _{(s, t)}\left|u_{N}(r)\right|_{H}^{2}\right]+\mathbb{E} \int_{s}^{t}\left\|u_{N}\right\|_{V}^{2} d r \leq & 2 \mathbb{E}\left|u_{0}\right|_{H}^{2}+2 \int_{0}^{t}\|f\|_{V^{\prime}}^{2} d s \\
& +2 \sigma\left(1+\sigma C_{1}^{2}\right)(t-s) .
\end{aligned}
$$

where $\sigma$ is the variance of $B$ and $C_{1}$ is a universal constant.

Remark 2.6. It can be noticed that, as in [4], the complete local energy inequality

$$
\begin{aligned}
\int_{D} \mid u(t) & \left.\right|^{2} \phi+2 \int_{0}^{t} \int_{D}|\nabla v|^{2} \phi \leq \\
\leq & \int_{0}^{t} \int_{D}|v|^{2}\left(\partial_{t} \phi+\triangle \phi\right)+2 \int_{0}^{t} \int_{D} \pi(v \cdot \nabla \phi) \\
& +\int_{0}^{t} \int_{D}\left(|v|^{2}+2 v \cdot z\right)((v+z) \cdot \nabla \phi)+2 \int_{0}^{t} \int_{D} \phi z \cdot((v+z) \cdot \nabla) v
\end{aligned}
$$

can be recovered using a cut-off function $\chi$ :

$$
0 \leq \chi \leq 1, \quad \chi=0 \text { for } s \leq 0, \quad \chi=1 \text { for } s \geq 1 ;
$$

for each $t$ we use $\phi_{\varepsilon}(x, s)=\phi(x, s) \chi\left(\frac{t-s}{\varepsilon}\right)$ as a test function and, as $\varepsilon \rightarrow 0$, we obtain the full local energy inequality.

Remark 2.7. The definition of suitable weak solution we have given seems to depend on the solution $z$ of the linear problem. This is not true, the definition given above has been introduced only to deal with the term $\partial_{t} g$. Indeed, it is possible to show the following result, which will be proved in Section 3

Theorem 2.8. The property of being a suitable weak solution for a pair $(u, P)$ does not depend on the solution $(z, Q)$ chosen for the linear problem.

The previous theorem tells us that, when $g \equiv 0$, there is no difference between the suitable weak solutions in the sense of Caffarelli, Kohn and Nirenberg [4] and ours. 
2.3. Stationary solutions. The approach we follow here concerning the framework of the path space and the introduction of stationary solutions is due to Sell [19] (see also [9]) and gives a solution to the problem of studying the asymptotic behaviour of dynamics when the dynamic itself cannot be well defined, as for Navier-Stokes equations.

A stationary solution is a measure on the space of all trajectories $(u, W)$ that are solutions to Navier-Stokes equations, which is invariant for the time-shift. In this setting we will not consider the pressure term $P$ explicitly, since we are mainly interested in the statistical properties of the velocity. In fact in [8] a regularity criterion will be proved which involves only the gradient of the velocity.

In order to have an equation whose deterministic part is autonomous, we will suppose that the deterministic forcing term $f \in L^{2}(D)$ is independent of time. The time-shift will act on the increments of the Brownian motion in order to preserve the stationarity of its increments.

Let $C_{0}([0,+\infty), H)$ be the set of all continuous functions which take value 0 in $t=0$ and let $S$ be the subset of $L_{\text {loc }}^{2}(0,+\infty ; H) \times C_{0}([0,+\infty), H)$ of all suitable weak solutions in $(0, \infty) \times D$, that is the set of all pairs $(u, W)$, where $W \in C^{1 / 2-\varepsilon}\left([0, T] ; D\left(A^{\beta}\right)\right)$ for $0<\varepsilon<\beta \leq \delta$, and $u$ is a suitable weak solution in the sense of Definition 2.3 for all $T>0$ under the body force $f+\partial_{t} W$. In this setting the pressure $P$ is treated as an auxiliary scalar field. We will see that this set is not empty. Let us define a metric on $\mathcal{S}$. Let

$$
\begin{aligned}
d_{1}\left(u^{1}, u^{2}\right) & =\sum_{n=1}^{\infty} 2^{-n}\left(1 \wedge \int_{0}^{n}\left|v^{1}-v^{2}\right|^{2} d t\right)^{\frac{1}{2}}, \\
d_{2}\left(W^{1}, W^{2}\right) & =\sum_{n=1}^{\infty} 2^{-n}\left(1 \wedge \sup _{(0, n)}\left|W^{1}-W^{2}\right|\right),
\end{aligned}
$$

and the metric on $\mathcal{S}$ is defined as

$$
d\left(\left(u^{1}, W^{1}\right),\left(u^{2}, W^{2}\right)\right)=d_{1}\left(u^{1}, u^{2}\right)+d_{2}\left(W^{1}, W^{2}\right) .
$$

Let $C_{b}(S)$ be the space of all bounded real continuous functions on $\mathcal{S}$ with the uniform topology, let $\mathcal{B}$ be the Borel $\sigma$-algebra of $(\mathcal{S}, d)$ and $M_{1}(\mathcal{S})$ be the set of all probability measures on $(\mathcal{S}, \mathcal{B})$.

Let $\tau_{t}: \mathcal{S} \rightarrow \mathcal{S},(t \geq 0)$ be the time shift on $\mathcal{S}$, defined as

$$
\tau_{t}(u, W)(s)=(u(s+t), W(t+s)-W(t)) .
$$

Notice that the map $(t, u, W) \rightarrow \tau_{t}(u, W)$ is continuous from $[0, \infty) \times \mathcal{S}$ to $\mathcal{S}$. We denote again by $\tau_{t}$ the induced mapping on $C_{b}(S)$, defined as

$$
\tau_{t} \phi(u)=\phi\left(\tau_{t} u\right)
$$

and by $\tau_{t} \mu$ the image measure of any $\mu \in M_{1}(\mathcal{S})$ under $\tau_{t}$, in the sense that

$$
\left\langle\tau_{t} \mu, \phi\right\rangle=\left\langle\mu, \tau_{t} \phi\right\rangle
$$

for each $\phi \in C_{b}(S)$. 
Definition 2.9. A probability measure $\mu \in M_{1}(S)$ is time-stationary if $\tau_{t} \mu=$ $\mu$ for all $t \geq 0$. A probability measure $\mu$ has finite mean dissipation rate if

$$
\int_{\mathcal{S}}\left[\int_{0}^{T} \int_{D}|\nabla u|^{2} d x d t\right] \mu(d u)<\infty
$$

for all $T>0$.

Remark 2.10. The property of having a finite dissipation rate is exactly the one we will need to apply the regularity criterion presented in [8]. Notice that this property does not depend on the presence of the noise, since there exist stationary solutions with finite dissipation rate also for the deterministic solution (see [7]).

Theorem 2.11. Let $f \in L^{2}(D)$ be independent of time. There exists a time stationary probability measure $\mu \in M_{1}(\mathcal{S})$ with finite mean dissipation rate. Moreover, there exists a constant $C_{\mu}>0$ such that for all $t \geq s \geq 0$,

$$
\int_{\mathcal{S}}\left[\int_{0}^{T} \int_{D}|\nabla u|^{2} d x d t\right] \mu(d u)=C_{\mu}(t-s) .
$$

Finally, the image measure of $\mu$ under the projection onto the second component is a Wiener measure whose covariance operator maps $H$ in $D\left(A^{\delta}\right)$, for a small $\delta>0$.

The last claim of the theorem says poorly that the standard process on $\mathcal{S}$ having law $\mu$ is a martingale suitable weak solution driven by a Brownian motion satisfying assumption (As).

\section{THE PROOF OF THEOREM 2.8}

Let $(u, P)$ be a suitable weak solution in the sense of Definition 2.3, that is, with respect to the solution $(z, Q)$ of problem (2.2). Let $\left(z_{1}, Q_{1}\right)$ be the solution of the equation

$$
\left\{\begin{array}{l}
\partial_{t} z_{1}-\triangle z_{1}+\nabla Q_{1}=f_{1} \\
\operatorname{div} z_{1}=0
\end{array}\right.
$$

with initial condition $z_{1}(0)=z_{0}$, and set

$$
w=z-z_{1} \quad \text { and } \quad R=Q-Q_{1} .
$$

Then $v_{1}=v+w, \pi_{1}=R+\pi$ and $v+z=v_{1}+z_{1}=u$. We show that $(u, P)$ is a suitable weak solution with respect to $\left(z_{1}, Q_{1}\right)$. In order to do this, we have only to show that $v_{1}$ satisfies the local energy inequality (3.3) (which has an additional term which takes into account the term $f-f_{1}$ ).

The function $w$ is the solution of

$$
\left\{\begin{array}{l}
\partial_{t} w-\Delta w+\nabla R=f_{2} \\
\operatorname{div} w=0 \\
w(0)=-z_{0}
\end{array}\right.
$$


where $f_{2}=f-f_{1}$. It is easy to see, by mollification, that for any $\phi \in$ $C_{c}^{\infty}\left(D_{T}\right), \phi \geq 0$,

$2 \int_{0}^{T} \int_{D}|\nabla w|^{2} \phi=\int_{0}^{T} \int_{D}|w|^{2}\left(\partial_{t} \phi+\triangle \phi\right)+2 \int_{0}^{T} \int_{D} R(w \cdot \nabla \phi)+2 \int_{0}^{T} \int_{D} \phi f_{2} \cdot w$.

Lemma 3.1. With the notations above, for any $\phi \in C_{c}^{\infty}\left(D_{T}\right)$,

$$
\begin{aligned}
4 \int_{0}^{T} \int_{D} \phi & \nabla v \cdot \nabla w= \\
= & 2 \int_{0}^{T} \int_{D} v \cdot w\left(\partial_{t} \phi+\triangle \phi\right)+2 \int_{0}^{T} \int_{D} R(v \cdot \nabla \phi)+2 \int_{0}^{T} \int_{D} \pi(w \cdot \nabla \phi) \\
& +2 \int_{0}^{T} \int_{D} \phi f_{2} \cdot v+\int_{0}^{T} \int_{D}\left(2 w \cdot z-|w|^{2}\right)((v+z) \cdot \nabla \phi) \\
& +2 \int_{0}^{T} \int_{D} \phi z \cdot((v+z) \cdot \nabla) w-2 \int_{0}^{T} \int_{D} \phi w \cdot((v+z) \cdot \nabla) v_{1}
\end{aligned}
$$

Proof. Let $\phi \in C_{c}^{\infty}\left(D_{T}\right)$; by mollification in a neighbourhood $U$ of Supp $\phi$ we obtain $\left(w_{\varepsilon}, R_{\varepsilon}\right)$ such that $w_{\varepsilon} \rightarrow w$ in $L^{\infty}\left(L^{2}\right), \nabla w_{\varepsilon} \rightarrow \nabla w$ in $L^{2}$ and $R_{\varepsilon} \rightarrow$ $R$ in $L^{5 / 3}$ in $U$.

Since $(v, \pi)$ is a weak solution of (2.3), we use $\phi w_{\varepsilon}$ as a test function to have

$$
\begin{array}{r}
\int_{0}^{T} \int_{D} v \cdot w_{\varepsilon} \partial_{t} \phi+\int_{0}^{T} \int_{D} \phi v \cdot \partial_{t} w_{\varepsilon}+\int_{0}^{T} \int_{D}(v+z) \cdot[(v+z) \cdot \nabla]\left(\phi w_{\varepsilon}\right)+ \\
+\int_{0}^{T} \int_{D} \pi w_{\varepsilon} \cdot \nabla \phi=\int_{0}^{T} \int_{D} \nabla v \cdot \nabla\left(\phi w_{\varepsilon}\right)
\end{array}
$$

Moreover we have in Supp $\phi$

$$
\left\{\begin{array}{l}
\partial_{t} w_{\varepsilon}-\Delta w_{\varepsilon}+\nabla R_{\varepsilon}=f_{2}, \\
\operatorname{div} w_{\varepsilon}=0
\end{array}\right.
$$

and multiplying by $\phi v$ and integrating by parts gives

$$
\int_{0}^{T} \int_{D} \phi v \cdot \partial_{t} w_{\varepsilon}+\int_{0}^{T} \int_{D} \nabla w_{\varepsilon} \cdot \nabla(\phi v)=\int_{0}^{T} \int_{D} R_{\varepsilon}(v \cdot \nabla \phi)+\int_{0}^{T} \int_{D} \phi f_{2} \cdot v \cdot
$$

Then we subtract (3.2) from (3.1) and we use the following facts (they can be easily obtained by integration by parts)

$$
\begin{aligned}
\int_{0}^{T} \int_{D} \nabla v \cdot \nabla\left(\phi w_{\varepsilon}\right)+\nabla w_{\varepsilon} \cdot \nabla(\phi v)= & \int_{0}^{T} \int_{D} 2 \phi \nabla v \cdot \nabla w_{\varepsilon}-v \cdot w_{\varepsilon} \triangle \phi, \\
\int_{0}^{T} \int_{D} \phi v((v+z) \cdot \nabla) w_{\varepsilon}= & -\int_{0}^{T} \int_{D} \phi w_{\varepsilon}((v+z) \cdot \nabla) v+ \\
& -\int_{0}^{T} \int_{D}\left(v \cdot w_{\varepsilon}\right)((v+z) \cdot \nabla \phi) \\
2 \int_{0}^{T} \int_{D} \phi w_{\varepsilon}((v+z) \cdot \nabla) w_{\varepsilon}= & -\int_{0}^{T} \int_{D}\left|w_{\varepsilon}\right|^{2}(v+z) \cdot \nabla \phi,
\end{aligned}
$$


so that we finally have

$$
\begin{aligned}
& 4 \int_{0}^{T} \int_{D} \phi \nabla v \cdot \nabla w_{\varepsilon}= \\
& \quad 2 \int_{0}^{T} \int_{D} v \cdot w_{\varepsilon}\left(\partial_{t} \phi+\triangle \phi\right)+2 \int_{0}^{T} \int_{D} R_{\varepsilon} v \cdot \nabla \phi+2 \int_{0}^{T} \int_{D} \pi w_{\varepsilon} \cdot \nabla \phi \\
& \quad+2 \int_{0}^{T} \int_{D} \phi v \cdot f_{2}+\int_{0}^{T} \int_{D}\left(2 z \cdot w_{\varepsilon}-\left|w_{\varepsilon}\right|^{2}\right)[(v+z) \cdot \nabla \phi] \\
& \quad+2 \int_{0}^{T} \int_{D} \phi z[(v+z) \cdot \nabla] w_{\varepsilon}-2 \int_{0}^{T} \int_{D} \phi w_{\varepsilon}[(v+z) \cdot \nabla]\left(v+w_{\varepsilon}\right)
\end{aligned}
$$

and, as $\varepsilon$ goes to 0 , the conclusion follows.

With the help of the above lemma, we can conclude the proof of Theorem 2.8. Let $\phi \in C_{c}^{\infty}\left(D_{T}\right)$, with $\phi \geq 0$, and $t \in(0, T]$. By definition

$$
2 \int_{0}^{T} \int_{D} \phi\left|\nabla v_{1}\right|^{2}=2 \int_{0}^{T} \int_{D} \phi|\nabla v|^{2}+2 \int_{0}^{T} \int_{D} \phi|\nabla w|^{2}+4 \int_{0}^{T} \int_{D} \phi \nabla v \cdot \nabla w .
$$

Since $(v, \pi)$ satisfies the energy inequality and using the energy equality for $(w, R)$ and the previous lemma, we have

$$
\begin{aligned}
2 \int_{0}^{T} \int_{D} \phi\left|\nabla v_{1}\right|^{2} \leq \\
\leq \int_{0}^{T} \int_{D}\left(|v|^{2}+2 v \cdot w+|w|^{2}\right)\left(\partial_{t} \phi+\triangle \phi\right)+2 \int_{0}^{T} \int_{D}(\pi+R)(v \cdot \nabla \phi) \\
\quad+\int_{0}^{T} \int_{D}\left(|v|^{2}+2 v \cdot z+2 w \cdot z-|w|^{2}\right)((v+z) \cdot \nabla \phi) \\
\quad+2 \int_{0}^{T} \int_{D} \phi z \cdot((v+z) \cdot \nabla)(v+w)+2 \int_{0}^{T} \int_{D}(R+\pi)(w \cdot \nabla \phi) \\
\quad+2 \int_{0}^{T} \int_{D} \phi f_{2} \cdot(v+w)-2 \int_{0}^{T} \int_{D} \phi w \cdot((v+z) \cdot \nabla) v_{1}
\end{aligned}
$$

Now we use the fact that $v_{1}=v+w, \pi_{1}=\pi+R, v+z=v_{1}+z_{1}$ and that

$$
|v|^{2}+2 v \cdot z+2 w \cdot z-|w|^{2}=\left|v_{1}\right|^{2}+2 v_{1} \cdot z_{1}
$$

to obtain the local energy inequality for $v_{1}$

$$
\begin{aligned}
2 \int_{0}^{T} \int_{D} \phi\left|\nabla v_{1}\right|^{2} \leq & \int_{0}^{T} \int_{D}\left|v_{1}\right|^{2}\left(\partial_{t} \phi+\triangle \phi\right)+2 \int_{0}^{T} \int_{D} \pi_{1}\left(v_{1} \cdot \nabla \phi\right) \\
& +\int_{0}^{T} \int_{D}\left(\left|v_{1}\right|^{2}+2 v_{1} \cdot z_{1}\right)\left(\left(v_{1}+z_{1}\right) \cdot \nabla \phi\right) \\
& +2 \int_{0}^{T} \int_{D} \phi z_{1} \cdot\left(\left(v_{1}+z_{1}\right) \cdot \nabla\right) v_{1}+2 \int_{0}^{T} \int_{D} \phi v_{1} \cdot f_{2} .
\end{aligned}
$$




\section{Proofs of THE EXISTENCE THEOREMS}

In this section we will prove Theorem 2.5, on existence for martingale suitable weak solutions, and Theorem 2.11, about the existence of stationary solutions. Prior to do this, we show a path-wise existence result, which will be the basis of the proofs of the two main theorems.

4.1. Path-wise existence. In this section it will be proved the existence of suitable weak solutions as defined in Definition 2.3. In other words we will show the following theorem

Theorem 4.1. Assume (Ad). There exists a suitable weak solution in the sense of Definition 2.3

The proof of this theorem is given in three steps. In the first step we solve a linearised version of the equation, whose higher regularity will let us prove the local energy inequality for such solutions. The second step will consist in the application of the Banach fixed point theorem to get the solution of an approximated nonlinear problem. In the third step we will find, in the limit of the approximation, a solution as requested by Theorem 4.1 .

We start with the first step.

Lemma 4.2. Let $u_{0} \in V, w \in L^{\infty}\left(D_{T}\right) \cap L^{2}(0, T ; V)$ and $\xi \in L^{2}(0, T ; V) \cap$ $L^{\infty}(0, T ; H)$. Then there exists a unique solution $(u, p)$ of the problem

$$
\begin{cases}\partial_{t} u-\triangle u+\nabla p+(w \cdot \nabla)(u+\xi)=0, & \\ \operatorname{div} u=0, & \text { on }(0, T) \times \partial D, \\ u=0 & \end{cases}
$$

with $u \in C\left([0, T] ; H_{0}^{1}(D)\right) \cap L^{2}\left(0, T ; H^{2}(D)\right)$ and $p \in L^{2}\left(0, T ; H^{1}(D)\right)$.

Moreover we have $\partial_{t} u \in L^{2}\left(D_{T}\right)$ and for any $\phi \in C_{c}^{\infty}\left(D_{T}\right), \phi \geq 0$,

$$
\begin{aligned}
2 \int_{0}^{T} \int_{D}|\nabla u|^{2} \phi= & \int_{0}^{T} \int_{D}|u|^{2}\left(\partial_{t} \phi+\triangle \phi\right)+2 \int_{0}^{T} \int_{D} p(u \cdot \nabla \phi) \\
& +2 \int_{0}^{T} \int_{D}\left(|u|^{2}+2 u \cdot \xi\right)(w \cdot \nabla \phi)+2 \int_{0}^{T} \int_{D} \phi \xi \cdot(w \cdot \nabla) u
\end{aligned}
$$

Proof. Let $\mathcal{B}: L^{2}(0, T ; V) \rightarrow L^{2}\left(0, T ; L^{2}(D)\right)$ be the operator

$$
\mathcal{B} u=(w \cdot \nabla) u
$$

It is easy to see that $(\mathcal{B} u, v)_{L^{2}(D)}=-(\mathcal{B} v, u)_{L^{2}(D)}$ for $u, v \in V$ and so it is easy to deduce that $(\mathcal{B} u, u)_{L^{2}(D)}=0$.

We look for a solution $u \in L^{2}(0, T ; V)$ of the problem

$$
\left\{\begin{array}{l}
\frac{d}{d t}(u, v)_{H}+(u, v)_{V}+(\mathcal{B}(u+\xi), v)_{L^{2}(D)}=0 \quad \text { for each } v \in V \\
u(0)=u_{0}
\end{array}\right.
$$


We will see that $u \in V \cap H^{2}$, then $A u \in H$ and so $u^{\prime}=-A u-P B(u+\xi)$ in $\mathrm{H}$ and $u^{\prime} \in L^{2}(0, T ; H)$, i.e. $u$ is equal a.e. to a continuous function from $[0, T]$ to $H$ (see Temam [22], Lemma 3.1.1) and the initial condition makes sense.

We prove existence by means of the Galerkin method. Let $v_{1}, \ldots, v_{m}, \ldots$ be a basis as above. We define for each $m \geq 1$ the approximate solution $u_{m}$ of the problem as follows

$$
u_{m}=\sum_{i=1}^{m} u_{m}^{i}(t) v_{i}
$$

and

$$
\left\{\begin{array}{l}
\left(u_{m}^{\prime}, v_{j}\right)_{H}+\left(u_{m}, v_{j}\right)_{V}+\left(\mathcal{B}\left(u_{m}+\xi\right), v_{j}\right)_{L^{2}(D)}=0 \quad j=1, \ldots, m \\
u_{m}(0)=u_{m}^{0}
\end{array}\right.
$$

where $u_{m}^{0}$ is the orthogonal projection in $H$ of $u_{0}$ on the linear space spanned by $v_{1}, \ldots, v_{m}$. This finite-dimensional linear system has a unique solution.

First we obtain an estimate of $u_{m}$ in $L^{\infty}(0, T ; H) \cap L^{2}(0, T ; V)$. Multiply each equation respectively by $u_{m}^{i}$ and sum to have

$$
\frac{d}{d t}\left|u_{m}\right|_{H}^{2}+\left.2|| u_{m}\right|_{V} ^{2}+2\left(\mathcal{B}\left(u_{m}+\xi\right), u_{m}\right)_{L^{2}(D)}=0
$$

Since

$$
\begin{aligned}
2\left(\mathcal{B}\left(u_{m}+\xi\right), u_{m}\right)_{L^{2}(D)} & =-2\left(\mathcal{B} u_{m}, \xi\right)_{L^{2}(D)} \\
& \leq\left\|u_{m}\right\|_{V}^{2}+\int_{D}|\xi|^{2}|w|^{2} \\
& \leq\left\|u_{m}\right\|_{V}^{2}+\|w\|_{L^{\infty}(D)}^{2}\|\xi\|_{L^{2}(D)}^{2}
\end{aligned}
$$

we have

$$
\frac{d}{d t}\left|u_{m}\right|_{H}^{2}+\left\|u_{m}\right\|_{V}^{2} \leq\|w\|_{L^{\infty}(D)}^{2}\|\xi\|_{L^{2}(D)}^{2}
$$

and consequently, integrating in time,

$$
\sup _{(0, T)}\left|u_{m}\right|_{H}^{2} \leq\left|u_{m}^{0}\right|_{H}^{2}+T\|w\|_{L^{\infty}\left(D_{T}\right)}^{2}\|\xi\|_{L^{\infty}\left(L^{2}(D)\right)}^{2}
$$

and

$$
\int_{0}^{T}\left\|u_{m}\right\|_{V}^{2} \leq\left|u_{m}^{0}\right|_{H}^{2}+T\|w\|_{L^{\infty}\left(D_{T}\right)}^{2}\|\xi\|_{L^{\infty}\left(L^{2}(D)\right)}^{2} .
$$

Then we obtain an estimate of $u_{m}$ in $L^{\infty}(0, T ; V) \cap L^{2}\left(0, T ; H^{2}(D)\right)$ and of $u_{m}^{\prime}$ in $L^{2}(0, T ; H)$. Multiply each equation by $u_{m}^{i}$ and sum to obtain

$$
\left|u_{m}^{\prime}\right|_{H}^{2}+\frac{1}{2} \frac{d}{d t}\left\|u_{m}\right\|_{V}^{2}+\left(\mathcal{B}\left(u_{m}+\xi\right), u_{m}^{\prime}\right)_{L^{2}(D)}=0,
$$

and so, by using Cauchy inequality and Young inequality,

$$
\left|u_{m}^{\prime}\right|_{H}^{2}+\frac{1}{2} \frac{d}{d t}|| u_{m}||_{V}^{2} \leq \frac{1}{2}\left|u_{m}^{\prime}\right|_{H}^{2}+\frac{1}{2} \int_{D}|w|^{2}\left|\nabla u_{m}+\nabla \xi\right|^{2}
$$


that is

$$
\left|u_{m}^{\prime}\right|_{H}^{2}+\frac{d}{d t} \|\left. u_{m}\right|_{V} ^{2} \leq \int_{D}|w|^{2}\left|\nabla u_{m}+\nabla \xi\right|^{2}
$$

in particular

$$
\frac{d}{d t}\left\|u_{m}\right\|_{V}^{2} \leq \int_{D}|w|^{2}\left|\nabla u_{m}+\nabla z\right|^{2} \leq 2\|w\|_{L^{\infty}(D)}^{2}\left\|u_{m}\right\|_{V}^{2}+2\|w\|_{L^{\infty}(D)}^{2}\|\xi\|_{V}^{2} .
$$

By Gronwall lemma we get

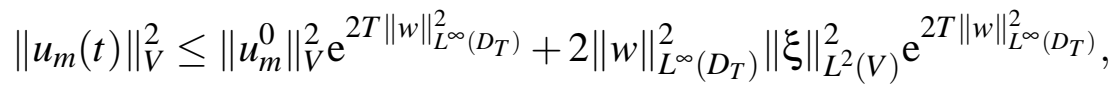

and, by integration by time, then we have

$$
\int_{0}^{T}\left|u_{m}^{\prime}\right|_{H}^{2} \leq\left\|u_{0}\right\|_{V}^{2}+2\|w\|_{L^{\infty}\left(D_{T}\right)}^{2}\left(\left\|u_{m}\right\|_{L^{\infty}(V)}^{2}+\|\xi\|_{L^{2}(V)}^{2}\right) .
$$

In conclusion we obtain that $u_{m}$ is bounded in $L^{\infty}(0, T ; V)$ and $u_{m}^{\prime}$ is bounded in $L^{2}(0, T ; H)$. From the equation then we get

$$
A u_{m}=-u_{m}^{\prime}-\mathcal{P} B\left(u_{m}+\xi\right)
$$

and so $A u_{m} \in L^{2}(0, T ; H)$; by the regularity theory for the Stokes operator, we obtain a bound for $u_{m}$ in the space $L^{2}\left(0, T ; H^{2}(D)\right)$.

Then there exist a subsequence $\left(u_{m^{\prime}}\right)_{m^{\prime} \in \mathbf{N}}$ of $\left(u_{m}\right)_{m \in \mathbf{N}}$ and a function $u$ such that $u_{m^{\prime}}$ converges weakly to $u$ in $L^{2}(0, T ; V)$ and $L^{2}\left(0, T ; H^{2}(D)\right)$ and converges weakly* in $L^{\infty}(0, T ; V)$ and in $L^{\infty}(0, T ; H)$. Moreover $u_{m^{\prime}}^{\prime}$ converges weakly to $u^{\prime}$ in $L^{2}(0, T ; H)$.

Taking the limit in the equation gives

$$
\frac{d}{d t}(u, v)_{H}+(u, v)_{V}+(\mathcal{B}(u+\xi), v)_{L^{2}(D)}=0 \quad \text { for each } v \in V
$$

in the sense of distributions on $[0, T]$.

We can easily see that the solution is unique, that $u \in C([0, T] ; V)$ and that

$$
u^{\prime}+A u+\mathcal{P} \mathcal{B}(u+\xi)=0 \quad \text { in } H .
$$

This means that $\mathcal{P}\left(u^{\prime}+A u+B(u+\xi)\right)=0$. Since $u^{\prime}+A u+B(u+\xi) \in$ $L^{2}\left(D_{T}\right)$, there exists a function $p$ such that $\nabla p(t) \in L^{2}(D)$ for a.e. t, and

$$
\partial_{t} u-\triangle u+(w \cdot \nabla)(u+\xi)+\nabla p=0
$$

whence $\nabla p \in L^{2}\left(D_{T}\right)$. Normalising $p$ by imposing that $\int_{D} p d x=0$, we obtain $p \in L^{2}\left(0, T ; H^{1}(D)\right)$.

Now we prove the energy equality. Let $\phi \in C_{c}^{\infty}\left(D_{T}\right)$ and $G=-(w \cdot \nabla)(u+$ $\xi)$. Then

$$
\partial_{t} u-\triangle u+\nabla p=G
$$

we mollify in $\mathbf{R}^{4}$ this equation in order to obtain smooth functions $u_{m}, p_{m}$ and $G_{m}$ such that

$$
\left\{\begin{array}{l}
\partial_{t} u_{m}-\triangle u_{m}+\nabla p_{m}=G_{m} \\
\operatorname{div} u_{m}=0
\end{array}\right.
$$


in a neighbourhood of Supp $\phi$ and such that

$$
\begin{array}{ll}
u_{m} \rightarrow u & \text { in } L^{\infty}\left(L^{2}(D)\right), \\
\nabla u_{m} \rightarrow \nabla u & \text { in } L^{2}, \\
p_{m} \rightarrow p & \text { in } L^{2}, \\
G_{m} \rightarrow G & \text { in } L^{2} ;
\end{array}
$$

then we multiply by $u_{m} \phi$ and integrate by parts to have

$2 \iint\left|\nabla u_{m}\right|^{2} \phi=\iint\left|u_{m}\right|^{2}\left(\partial_{t} \phi+\triangle \phi\right)+2 \iint p_{m}\left(u_{m} \cdot \nabla \phi\right)+2 \iint\left(u_{m} \cdot G_{m}\right) \phi$.

As $m \rightarrow \infty$, we recover the energy equality, using the fact that $G=-(w$. $\nabla)(u+\xi)$ and by integration by parts.

In the second step of the proof of Theorem 4.1 we obtain the solution for the approximated nonlinear equation. We firstly define a regularisation procedure. Let $v_{1}, \ldots, v_{m}, \ldots$ be an orthonormal basis in $H$ of eigenfunctions of the operator $A$. For any $N \in \mathbf{N}$ and $v \in H$, we denote by $v^{N}$ the projection of $v$ on the span of $v_{1}, \ldots, v_{N}$. The following properties hold

1. $\left|v^{N}\right|_{H} \leq|v|_{H}$

2. $\left\|v^{N}\right\|_{V} \leq\|v\|_{V}$;

3. $\left\|v-v^{N}\right\|_{V} \rightarrow 0$ if $v \in V$.

Notice that, by virtue of assumption (Ad), we know that

$$
z \in L^{\infty}(0, T ; H) \cap L^{2}(0, T ; V) \cap L^{8+\varepsilon}\left(0, T ; L^{4}(D)\right) .
$$

for some $\varepsilon>0$. Actually we know much more, namely that $z$ is bounded with values in $L^{4}(D)$, but, as we shall see, a weaker bound (like the one given above) is sufficient.

Lemma 4.3. Assume (Ad). Let $N \in N$, then there exists a pair $\left(v_{N}, \pi_{N}\right)$, with $v_{N} \in L^{2}\left(0, T ; H^{2}(D)\right) \cap C\left([0, T] ; H_{0}^{1}(D)\right)$ and $\partial_{t} v_{N} \in L^{2}\left(D_{T}\right), \pi_{N} \in L^{2}\left(D_{T}\right)$ with $\int_{D} \pi_{N}=0$, that solves the following equation

$$
\partial_{t} v_{N}-\triangle v_{N}+\left[\left(\left(v_{N}\right)^{N}+z^{N}\right) \cdot \nabla\right]\left(v_{N}+z\right)+\nabla \pi_{N}=0
$$

with initial condition $v_{N}(0)=u_{0}^{N}$, and such that for any $\phi \in C_{c}^{\infty}\left(D_{T}\right), \phi \geq 0$, the following energy equality holds

$$
\begin{aligned}
2 \int_{0}^{T} \int_{D}\left|\nabla v_{N}\right|^{2} \phi= & \int_{0}^{T} \int_{D}\left|v_{N}\right|^{2}\left(\partial_{t} \phi+\triangle \phi\right)+2 \int_{0}^{T} \int_{D} \pi_{N}\left(v_{N} \cdot \nabla \phi\right) \\
& +\int_{0}^{T} \int_{D}\left(\left|v_{N}\right|^{2}+2 v_{N} \cdot z\right)\left(\left(\left(v_{N}\right)^{N}+z^{N}\right) \cdot \nabla \phi\right) \\
& +2 \int_{0}^{T} \int_{D} \phi z \cdot\left(\left(\left(v_{N}\right)^{N}+z^{N}\right) \cdot \nabla\right) v_{N}
\end{aligned}
$$

Proof. Fix $N \in \mathbf{N}$ and let

$$
\mathcal{C}=\left\{w \in L^{2}(0, T ; V) \cap L^{\infty}(0, T ; H) \mid\|w\|_{\mathcal{C}} \leq R_{0}\right\},
$$


where

$$
\|\cdot\|_{C}=\left(\|\cdot\|_{L^{2}(V)}^{2}+\|\cdot\|_{L^{\infty}(H)}^{2}\right)^{1 / 2}
$$

and $R_{0}$ will be fixed later. Define a function $\mathcal{F}$ from $\mathcal{C}$ to $L^{\infty}(0, T ; H) \cap$ $L^{2}(0, T ; V)$ as follows: if $w \in \mathcal{C}$, we take the regularisation $w^{N}$ as above and $u=\mathcal{F} w$ is the solution of the problem

1. $\partial_{t} u-\triangle u+\left[\left(w^{N}+z^{N}\right) \cdot \nabla\right](u+z)+\nabla p=0$,

2. $\operatorname{div} u=0$,

3. $u(0)=u_{0}^{N}$,

4. $u \in L^{2}\left(0, T ; H^{2}(D)\right) \cap C\left([0, T] ; H_{0}^{1}(D)\right)$,

5. $\partial_{t} u, p \in L^{2}\left(D_{T}\right)$ and $\int_{D} p d x=0$,

6. for any $\phi \in C_{c}^{\infty}\left(D_{T}\right), \phi \geq 0$,

$$
\begin{aligned}
2 \int_{0}^{T} \int_{D}|\nabla u|^{2} \phi= & \int_{0}^{T} \int_{D}|u|^{2}\left(\partial_{t} \phi+\triangle \phi\right)+2 \int_{0}^{T} \int_{D} p(u \cdot \nabla \phi) \\
& +\int_{0}^{T} \int_{D}\left(|u|^{2}+2 u \cdot z\right)\left(\left(w^{N}+z^{N}\right) \cdot \nabla \phi\right) \\
& +2 \int_{0}^{T} \int_{D} \phi z \cdot\left(\left(w^{N}+z^{N}\right) \cdot \nabla\right) u
\end{aligned}
$$

The existence and uniqueness of this solution is guaranteed by the previous lemma, once we apply it with $w \rightarrow w^{N}+z^{N}$ and $\xi \rightarrow z$.

First we show that $\mathcal{F}$ maps $C$ into itself. In order to show this, we shall only choose a suitable $R_{0}$. We have

$$
\begin{aligned}
\|\mathcal{F} w\|_{L^{\infty}(H)}^{2}+2\|\mathcal{F} w\|_{L^{2}(V)}^{2} & \leq\left|u_{0}^{N}\right|^{2}+2 \int_{0}^{T} \int_{D}|z| \cdot\left|w^{N}+z^{N}\right| \cdot|\nabla \mathcal{F} w| \\
& \leq\left|u_{0}\right|^{2}+\|\mathcal{F} w\|_{L^{2}(V)}^{2}+\int_{0}^{T} \int_{D}|z|^{2}\left|w^{N}+z^{N}\right|^{2}
\end{aligned}
$$

and so, since in finite-dimensional spaces all the norms are equivalent, we have

$$
\left\|w^{N}+z^{N}\right\|_{L^{\infty}} \leq C_{N}\left\|w^{N}+z^{N}\right\|_{L^{\infty}(H)} \leq C_{N}\|w+z\|_{L^{\infty}(H)} .
$$

Then

$$
\begin{aligned}
\|\mathcal{F} w\|_{L^{\infty}(H)}^{2}+2\|\mathcal{F} w\|_{L^{2}(V)}^{2} & \leq\left|u_{0}\right|_{H}^{2}+C_{N}^{2} T\|z\|_{L^{\infty}(H)}^{2}\|w+z\|_{L^{\infty}(H)}^{2} \\
& \leq\left|u_{0}\right|_{H}^{2}+2 C_{N}^{2} T\|z\|_{L^{\infty}(H)}^{2}\left(R_{0}^{2}+\|z\|_{L^{\infty}(H)}^{2}\right) \\
& \leq R_{0}^{2}
\end{aligned}
$$

if we choose $R_{0}>\left|u_{0}\right|^{2}$ and $T$ small enough.

Then we show that $\mathcal{F}$ is a contraction. Let $w_{1}, w_{2} \in \mathcal{C}$ and set $w=w_{1}-$ $w_{2}, v=\mathcal{F} w_{1}-\mathcal{F} w_{2}$ and $p=p_{1}-p_{2}$, where $p_{1}, p_{2}$ are the corresponding pressures. Then

$$
\partial_{t} v-\triangle v+\nabla p=-\left(w^{N} \cdot \nabla\right)\left(\mathcal{F} w_{1}+z\right)-\left[\left(w_{2}^{N}+z^{N}\right) \cdot \nabla\right] v,
$$


and so, using the fact that $\mathrm{v}(0)=0$, we have

$$
\begin{aligned}
\|v\|_{L^{\infty}(H)}^{2}+2\|v\|_{L^{2}(V)}^{2} & \leq 2 \int_{0}^{T} \int_{D}\left|\mathcal{F} w_{1}+z\right| \cdot\left|w^{N}\right| \cdot|\nabla v| \\
& \leq 2\left\|\mathcal{F} w_{1}+z\right\|_{L^{\infty}(H)} \cdot\left\|w^{N}\right\|_{L^{\infty}\left(D_{T}\right)} \int_{0}^{T}\|\nabla v\|_{L^{2}(D)} \\
& \leq 2 T^{\frac{1}{2}}\left\|\mathcal{F} w_{1}+z\right\|_{L^{\infty}(H)} \cdot\left\|w^{N}\right\|_{L^{\infty}\left(D_{T}\right)}\|\nabla v\|_{L^{2}\left(D_{T}\right)} .
\end{aligned}
$$

In particular

$$
\|v\|_{L^{2}(V)} \leq T^{1 / 2}\left\|\mathcal{F} w_{1}+z\right\|_{L^{\infty}(H)} \cdot\left\|w^{N}\right\|_{L^{\infty}\left(D_{T}\right)},
$$

and it follows that

$$
\begin{aligned}
\|v\|_{L^{\infty}(H)}^{2}+\|v\|_{L^{2}(V)}^{2} & \leq 2 T\left\|\mathcal{F} w_{1}+z\right\|_{L^{\infty}(H)}^{2} \cdot\left\|w^{N}\right\|_{L^{\infty}\left(D_{T}\right)}^{2} \\
& \leq 4 T C_{N}^{2}\left(R_{0}^{2}+\|z\|_{L^{\infty}(H)}^{2}\right)\|w\|_{C}^{2} .
\end{aligned}
$$

In conclusion

$$
\left\|\mathcal{F} w_{1}-\mathcal{F} w_{2}\right\|_{\mathcal{C}}^{2} \leq 4 T C_{N}^{2}\left(R_{0}^{2}+\|z\|_{L^{\infty}(H)}^{2}\right)\left\|w_{1}-w_{2}\right\|_{C}^{2}
$$

and, if we choose the time interval small enough, the map $\mathcal{F}$ is a contraction.

Then the last step of the proof follows. We show that the sequence $\left(v_{N}, \pi_{N}\right)$ converges to a weak solution $(v, \pi)$ satisfying the properties of Definition 2.3

Proof of Theorem 4.1 First we get an estimate of the solutions in the spaces $L^{\infty}(0, T ; H)$ and $L^{2}(0, T ; V)$. Indeed, multiply the equation by $v^{N}$ and integrate by parts to get

$$
\frac{1}{2} \frac{d}{d t}\left|v_{N}\right|_{H}^{2}+\left\|\nabla v_{N}\right\|_{L^{2}(D)}^{2}=\int_{D} z \cdot\left[\left(\left(v_{N}\right)^{N}+z^{N}\right) \cdot \nabla\right] v_{N}
$$

By using Hölder inequality and Young inequality we get

$$
\begin{aligned}
\int_{D} z \cdot & {\left.\left[\left(v_{N}\right)^{N}+z^{N}\right) \cdot \nabla\right] v_{N} \leq } \\
& \leq \int_{D}|z| \cdot\left|\nabla v_{N}\right| \cdot\left|\left(v_{N}\right)^{N}\right|+\int_{D}|z| \cdot\left|z^{N}\right| \cdot\left|\nabla v_{N}\right| \\
& \leq\left\|\nabla v_{N}\right\|_{L^{2}(D)}\left\|\left(v_{N}\right)^{N}\right\|_{L^{4}(D)}\|z\|_{L^{4}(D)}+\left\|\nabla v_{N}\right\|_{L^{2}(D)}\|z\|_{L^{4}(D)}\left\|z^{N}\right\|_{L^{4}(D)} \\
& \leq C^{2}\left\|\nabla v_{N}\right\|_{L^{2}(D)}^{7 / 4}\left|v_{N}\right|_{H}^{1 / 4}\|z\|_{L^{4}(D)}+C\left\|\nabla v_{N}\right\|_{L^{2}(D)}|z|_{H}^{1 / 4}\|z\|_{V}^{3 / 4} \\
& \leq \frac{1}{2}\left\|\nabla v_{N}\right\|_{L^{2}(D)}^{2}+C^{2}\|z\|_{L^{4}(D)}^{2}|z|_{H}^{1 / 2}\|z\|_{V}^{3 / 2}+\frac{7^{7}}{2^{10}} C^{16}\|z\|_{L^{4}(D)}^{8}\left|v_{N}\right|_{H}^{2}
\end{aligned}
$$

and so

$$
\frac{d}{d t}\left|v_{N}\right|^{2}+\left\|\nabla v_{N}\right\|^{2} \leq 2 C^{2}\|z\|_{L^{4}(D)}^{2}|z|^{1 / 2}\|z\|^{3 / 2}+\frac{7^{7}}{2^{9}} C^{16}\|z\|_{L^{4}(D)}^{8}\left|v_{N}\right|^{2}
$$


since, for any suitable $\xi$, by virtue of Sobolev inequalities,

$$
\left\|\xi^{N}\right\|_{L^{4}(D)} \leq C\left|\xi^{N}\right|_{H}^{1 / 4}\left\|\xi^{N}\right\|_{V}^{3 / 4} \leq C|\xi|_{H}^{1 / 4}\|\xi\|_{V}^{3 / 4}
$$

Then, by Gronwall lemma,

$$
\sup \left|v_{N}\right|_{H}^{2} \leq C(T, z)+\left|u_{0}^{N}\right|_{H}^{2} \leq\left|u_{0}\right|_{H}^{2}+C(T, z)
$$

and, integrating with respect to time,

$$
\int_{0}^{T}\left\|\nabla v_{N}\right\|_{L^{2}}^{2} \leq\left|u_{0}\right|_{H}^{2}+C(T, z)
$$

where $C(T, z)$ is a constant which depends only on $T$ and on the function $z$.

Then we give an estimate of the pressure term. By Theorem 15 of [20] we can deduce that $\nabla \pi_{N}$ are bounded in $L^{5 / 4}\left((\varepsilon, T) ; L^{5 / 4}(D)\right)$ for every $\varepsilon>0$. Then using the argument given in [4] (page 781), we can conclude that $\pi_{N}$ are bounded in $L^{5 / 4}\left((\varepsilon, T) ; L_{\text {loc }}^{5 / 3}(D)\right)$, provided that

$$
\int_{D} \pi_{N} d x=0
$$

at each time.

We can improve the regularity of $\pi_{N}$ using the general result of Sohr and Von Wahl [21] or the simplified argument of Lin [12], to obtain that $\pi_{N}$ are bounded in $L_{\text {loc }}^{5 / 3}((0, T] \times D)$.

At last, using an argument similar to the one in Lemma 4.2 (Chapter III) of [22], we know that $v_{N}$ are bounded in $W^{1,2}\left(0, T ; D\left(A^{-1}\right)\right)$ and so, by virtue of Theorem 2.1 (Chapter III) of [22], $\left(v_{N}\right)_{N \in \mathbf{N}}$ is compact in $L^{2}\left(D_{T}\right)$.

We can deduce then that there exist a subsequence of $\left(v_{N}, \pi_{N}\right)_{N \in \mathbf{N}}$, which we call again $\left(v_{N}, \pi_{N}\right)$, and functions $(v, \pi)$ such that

1. $v_{N} \rightarrow v$ weakly* in $L^{\infty}\left(0, T ; L^{2}(D)\right)$,

2. $\nabla v_{N} \rightarrow \nabla v$ weakly in $L^{2}\left(D_{T}\right)$,

3. $v_{N} \rightarrow v$ strongly in $L^{2}\left(D_{T}\right)$,

4. $\pi_{N} \rightarrow \pi$ weakly in $L_{\text {loc }}^{5 / 3}((0, T] \times D)$,

5. $v_{N}^{\prime}$ is bounded in $L^{2}\left(0, T ; D\left(A^{-1}\right)\right)$.

These convergence properties are sufficient to verify that the limit $v$ is a weak solution of Navier-Stokes system. Moreover the initial condition is satisfied, in fact $v_{N}$ are weakly continuous uniformly, by the bound of their derivatives, and so

$$
v(0)=\lim _{N} v_{N}(0)=u_{0} .
$$

At last, thanks to the uniform bound on the time derivative, the limit is continuous as a function from $[0, T]$ to the space $H$ with the weak topology.

Now we prove the classical energy inequality. Integrate 4.1) in time between $s$ and $t$, then in the limit as $N \rightarrow \infty$, the classical energy inequality for $v$ is obtained.

The last step of the proof is to prove that the limit $v$ verifies the local energy inequality. Since $v_{N} \rightarrow v$ in $L^{2}\left(D_{T}\right)$ and $v_{N}$ are bounded in $L^{10 / 3}\left(D_{T}\right)$ 
by Sobolev inequalities, then $v_{N} \rightarrow v$ in $L^{q}\left(D_{T}\right)$ for any $q \in\left[2, \frac{10}{3}\right)$. By the properties of the regularisation, we can deduce that $\left(v_{N}+z\right)^{N} \rightarrow(v+z)$ in $L^{2}\left(D_{T}\right)$ and, in the same way as above, in $L^{q}\left(D_{T}\right)$.

Let $\varphi \in C_{c}^{\infty}\left(D_{T}\right)$. We know that

$$
\begin{aligned}
\iint\left|\nabla v_{N}\right|^{2} \varphi= & \iint\left|v_{N}\right|^{2}\left(\partial_{t} \varphi+\triangle \varphi\right)+\iint 2 \pi_{N} v_{N} \cdot \nabla \varphi \\
& +\iint\left(\left|v_{N}\right|^{2}+2 v_{N} \cdot z\right)\left(\left(v_{N}+z\right)^{N} \cdot \nabla \varphi\right) \\
& +2 \iint \varphi z \cdot\left[\left(v_{N}+z\right)^{N} \cdot \nabla\right] v_{N} .
\end{aligned}
$$

By lower semi-continuity

$$
\iint|\nabla v|^{2} \varphi \leq \liminf \iint\left|\nabla v_{N}\right|^{2} \varphi
$$

moreover, since $v_{N}$ converges strongly in $L^{q}\left(D_{T}\right)$, with $q \in\left[2, \frac{10}{3}\right)$, and $p_{N}$ converges weakly in $L_{\mathrm{loc}}^{5 / 3}\left(D_{T}\right)$, the first three terms converge. In order to show that the last term also converges, we use the fact that $z$ is bounded in $L^{8+\varepsilon}\left(0, T ; L^{4}(D)\right)$, with $\varepsilon>0$ (this is the only step of the proof where we need this fact). Let

$$
q=\frac{6(8+\varepsilon)}{12+\varepsilon} \in(4,6), \quad p=\frac{4 q}{3 q-6} \in\left(2, \frac{8}{3}\right)
$$

then we know that $\left(v_{N}+z\right)^{N}$ converges in $L^{p}\left(D_{T}\right)$, by the previous considerations, and is bounded in $L^{p}\left(0, T ; L^{q}(D)\right)$ by the Sobolev inequality. Thus by interpolation $\left(v_{N}+z\right)^{N}$ converges in the space $L^{p}\left(0, T ; L^{4}(D)\right)$ and this is sufficient to conclude since

$$
\frac{1}{2}+\frac{1}{p}+\frac{1}{8+\varepsilon}=1
$$

4.2. The proof of Theorem 2.5. We can use now the results of the previous section to show the existence of martingale suitable weak solutions. There are some technical points in the proof of this theorem, mostly linked to the fact that we deal with a pair $(v, \pi)$ of processes, where we have no information on the tightness of the laws of the approximating sequence of pressures. We solve the problem by means of the following lemma, which is given in a generalised setting.

Let $\left(S, d_{S}\right)$ and $\left(T, d_{T}\right)$ be two complete separable metric spaces and consider the product metric space $S \times T$, endowed of the product metric. Let $\pi: S \times T \rightarrow S$ be the canonical projection onto the first component, that is $\pi(s, t)=s$ for $(s, t) \in S \times T$. Let a sequence of measure $\mathrm{v}_{n}$ be given on $S \times T$ such that

$$
\mu_{n}=\pi v_{n} \rightarrow \mu
$$

where $\mu$ is a measure on $S$. 
Lemma 4.4. There exist a probability space $(\Omega, \mathcal{F}, \mathbb{P})$, a sequence of random variables $\left(X_{n}, Y_{n}\right)$ on $S \times T$, the laws of which are $v_{n}$, and a random variable $X$ on $S$ having $\mu$ as its law, such that

$$
X_{n} \rightarrow X \quad \mathbb{P}-\text { a.s. }
$$

Proof. From Theoreme 1, §6, No. 1 of Bourbaki [3], since $T$ is a complete separable metric space, it may be homeomorphic-ally embedded as a $G_{\delta}$ subset (a countable intersection of open sets), and so as a Borel set, of a compact metrizable space $\tilde{T}$. So measures $v_{n}$ can be extended to measures $\tilde{\mathrm{v}}_{n}$ in the space $\tilde{T}$ : in this way the sequence $\left(\tilde{\mathrm{v}}_{n}\right)_{n \in \mathbf{N}}$ is tight and by Prohorov theorem there exists a subsequence, called again $\left(\tilde{v}_{n}\right)_{n \in \mathbf{N}}$, converging weakly to a measure $\tilde{\mathrm{v}}$ on $\tilde{T}$. Obviously $\tilde{\pi} \tilde{\mathrm{V}}_{n}=\mu_{n}$ and $\tilde{\pi} \tilde{\mathrm{v}}=\mu$, where $\tilde{\pi}$ is the projection of the space $S \times \tilde{T}$ onto the first component. By Skorohod theorem there exist a probability space $(\Omega, \mathcal{F}, \mathbb{P})$, random variables $\left(\tilde{X}_{n}, \tilde{Y}_{n}\right)$ on $S \times \tilde{T}$, with laws $\tilde{\mathrm{v}}_{n}$, and $(\tilde{X}, \tilde{Y})$, with law $\tilde{\mu}$, such that

$$
\left(\tilde{X}_{n}, \tilde{Y}_{n}\right) \rightarrow(\tilde{X}, \tilde{Y}) \quad \mathbb{P}-\text { a.s. }
$$

Since $\tilde{\mathrm{v}}_{n}(S \times T)=1$, the restrictions to the space $S \times T$ of the previous random variables $\left(X_{n}, Y_{n}\right)$ (notice that $\left.X_{n}=\tilde{X}_{n}\right)$, have $v_{n}$ as their laws. Moreover, $\tilde{X}$ has $\mu$ as its law and $X_{n} \rightarrow \tilde{X}$, P-a.s.

Remark 4.5. In [15] it is given an alternative proof of this fact, showing that actually the claim is true for the whole sequence, not only for a subsequence. Since we will use the lemma together with a compactness argument, we don't really need the complete result.

Proof of Theorem 2.5 Let $z$ be the stochastic process which is solution of system (2.2). We know that for a.e. $\omega \in \Omega$ we have that $W(\omega)$ is in the space $C^{1 / 2-\varepsilon}\left([0, T] ; D\left(A^{\delta}\right)\right)$ for all $\varepsilon<\delta$, and so by [6] we can deduce that $z(\omega)$ satisfies (2.4). So we can apply path-wise the results of the previous section. For a.e. $\omega \in \Omega$ we use Lemma 4.3 to get for each $N \in \mathbf{N}$ a pair $\left(v_{N}, \pi_{N}\right)$. The map

$$
\omega \in \Omega \mapsto\left(v_{N}(\omega), \pi_{N}(\omega), z(\omega), W(\omega)\right)
$$

with values in

$$
L^{2}(0, T ; H) \times L_{\text {loc }}^{5 / 3}\left(D_{T}\right) \times C([0, T] ; H) \times C_{0}([0, T] ; H)
$$

is measurable for any $T>0$. The random variable $v_{N}(t)$ is measurable for almost each $t \geq 0$ since each vector field is continuous with values in $H$. In fact, if $\eta_{\varepsilon}$ are mollifiers, then

$$
\eta_{\varepsilon} * v_{N}(t) \rightarrow v_{N}(t) \quad \text { in } H
$$

and $\eta_{\varepsilon} * v_{N}$ are measurable. In the same way, thanks to uniqueness of the solutions, we can use a smarter regularisation, namely

$$
v_{N}^{0}+\frac{1}{\varepsilon} \int_{0}^{t} \mathrm{e}^{\frac{s-t}{\varepsilon}}\left(v_{N}(s)-v_{N}^{0}\right) d s
$$

to show that $v_{N}$ is a progressively measurable process. 
So it is well defined a random variable $\left(v_{N}(\cdot, \omega), \pi_{N}(\cdot, \omega), z(\cdot, \omega), W(\cdot, \omega)\right)$ such that $z$ satisfies $(2.2),\left(v_{N}, \pi_{N}\right)$ is the solution of the approximated problem for almost each $\omega \in \Omega$, and $W$ is a Brownian motion.

Let $v_{N}$ be the law of $\left(v_{N}, \pi_{N}, z, W\right)$ in

$$
\mathcal{E}=L^{2}(0, T ; H) \times L_{\mathrm{loc}}^{5 / 3}\left(D_{T}\right) \times C([0, T] ; H) \times C_{0}([0, T] ; H)
$$

and let $\mu_{N}$ be the projection of $v_{N}$ in the variable $v$, that is the law of $v_{N}$. We want to show that the family of measures $\mu_{N}$ is tight, that is for each $\varepsilon>0$ there exists a compact set $K_{\varepsilon}$ in $\mathcal{E}$ such that

$$
\mu_{N}\left(K_{\varepsilon}\right) \geq 1-\varepsilon \quad N \in \mathbf{N} .
$$

We take

$$
K_{\varepsilon}=\left\{v \mid\|v\|_{L^{\infty}(0, T ; H)}^{2}+\|v\|_{L^{2}(0, T ; V)}^{2}+\|v\|_{H^{1}\left(0, T ; D\left(A^{-1}\right)\right)} \leq C_{\varepsilon}\right\} .
$$

The set $K_{\varepsilon}$ is compact in $L^{2}(0, T ; H)$ and, moreover,

$$
\mathbb{P}\left[v \notin K_{\varepsilon}\right] \leq \frac{1}{C_{\varepsilon}} \mathbb{E}\left(\left\|v_{N}\right\|_{L^{\infty}(0, T ; H)}^{2}+\left\|v_{N}\right\|_{L^{2}(0, T ; V)}^{2}+\left\|v_{N}\right\|_{H^{1}\left(0, T ; D\left(A^{-1}\right)\right)}\right)
$$

and the right hand side is smaller than $\varepsilon$ if the above mean values are uniformly bounded with respect to $N$ and $C_{\varepsilon}$ is chosen properly. To see this, fix $N \in \mathbf{N}$ and let $u_{N}=v_{N}+z$. First we have (this can be done as in Lemma 2.3 in [12])

$$
\left\|\partial_{t} v_{N}\right\|_{L^{2}\left(0, T ; D\left(A^{-1}\right)\right)} \leq\left\|v_{N}\right\|_{L^{2}(0, T ; V)}+\left\|u_{N}\right\|_{L^{2}(0, T ; H)}\left\|u_{N}\right\|_{L^{2}(0, T ; V)},
$$

moreover $\mathbb{E}\|z\|_{L^{2}(0, T ; V)}^{2}+\mathbb{E}\|z\|_{L^{\infty}(0, T ; H)}^{2}$ is finite and so the only thing we need to show is that

$$
\mathbb{E}\left\|u_{N}\right\|_{L^{\infty}(0, T ; H)}^{2}+\mathbb{E}\left\|u_{N}\right\|_{L^{2}(0, T ; V)}^{2}
$$

is bounded uniformly in $N$. We know that

$$
u_{N}(t)=v_{0}^{N}+\int_{0}^{t}\left(A u_{N}+\left(\left(u_{N}\right)^{N} \cdot \nabla\right) u_{N}+f^{N}\right) d s+W_{t}
$$

and so (see Pardoux [14], Théorème 3.1)

$$
\begin{aligned}
\left|u_{N}(t)\right|_{H}^{2}+ & 2 \int_{0}^{t}\left\|u_{N}\right\|_{V}^{2} d s= \\
& =\left|u_{0}^{N}\right|_{H}^{2}+2 \int_{0}^{t}\left\langle f^{N}, u_{N}\right\rangle_{H} d s+2 \int_{0}^{t}\left\langle u_{N}(s), d W_{s}\right\rangle_{H}+\sigma t .
\end{aligned}
$$

Notice that, if $\tau_{R}=\inf \left\{t>0|| u_{N}(t) \mid>R\right\}$, then

$$
\int_{0}^{t}\left\langle u_{N}(s), d W_{s}\right\rangle_{H}
$$


is a local martingale with respect to the stopping time $\tau_{R}$, and so, taking the expectation of (4.2) at time $t \wedge \tau_{R}$,

$$
\begin{aligned}
& \mathbb{E}\left|u_{N}\left(t \wedge \tau_{R}\right)\right|_{H}^{2}+2 \mathbb{E} \int_{0}^{t \wedge \tau_{R}}\left\|u_{N}\right\|_{V}^{2} d s= \\
& =\mathbb{E}\left|u_{0}^{N}\right|_{H}^{2}+\mathbb{E} \int_{0}^{t \wedge \tau_{R}}\left|f^{N}\right|_{H}^{2} d s+\mathbb{E} \int_{0}^{t \wedge \tau_{R}}\left|u_{N}\right|_{H}^{2} d s+\sigma t \wedge \tau_{R},
\end{aligned}
$$

Let $\varphi(t)=\mathbb{E}\left|u_{N}\left(t \wedge \tau_{R}\right)\right|_{H}^{2}$, then we have

$$
\varphi(t) \leq \varphi(0)+\int_{0}^{t}|f|_{H}^{2} d s+\int_{0}^{t} \varphi(s) d s+\sigma t
$$

and by Gronwall's lemma we can deduce that $\varphi(t)$ is bounded by a constant independent of $R$. So, as $R \uparrow \infty$, we can deduce that $\mathbb{E}\left|u^{N}(t)\right|_{H}^{2} \leq C(T)$, and then that 4.3) is a martingale.

So, by taking the expectation in (4.2), we obtain first that

$$
\mathbb{E}\left|u_{N}(t)\right|_{H}^{2}+\mathbb{E} \int_{s}^{t}\left\|u_{N}\right\|_{V}^{2} d r \leq \mathbb{E}\left|u_{N}(s)\right|_{H}^{2}+\sigma(t-s)+\mathbb{E} \int_{s}^{t}\left\|f^{N}\right\|_{V^{\prime}} d s
$$

and then, using the Burkholder-Davis-Gundy inequality, that

$$
\begin{aligned}
\mathbb{E}\left[\sup _{s \leq t}\left|u_{N}(s)\right|_{H}^{2}\right] & +\mathbb{E} \int_{0}^{t}\left\|u_{N}\right\|_{V}^{2} d s \leq \\
\leq & \mathbb{E}\left|u_{0}^{N}\right|_{H}^{2}+\int_{0}^{t}\left\|f^{N}\right\|_{V^{\prime}}^{2} d s+\sigma t+2 \sigma C_{1} \mathbb{E}\left[\int_{0}^{t}\left|u_{N}\right|_{H}^{2} d s\right]^{\frac{1}{2}} .
\end{aligned}
$$

We can conclude that

$$
\mathbb{E}\left[\sup _{s \leq t}\left|u_{N}(s)\right|^{2}\right]+\mathbb{E} \int_{0}^{t}\left\|u_{N}\right\|^{2} d s \leq 2 \mathbb{E}\left|u_{0}^{N}\right|^{2}+2 \int_{0}^{t}\left\|f^{N}\right\|_{V^{\prime}}^{2} d s+2 \sigma\left(1+\sigma C_{1}^{2}\right) t
$$

and the claim is proved.

By Lemma 4.4 there exist a probability space $(\tilde{\Omega}, \tilde{\mathcal{F}}, \mathbb{P})$ and random variables $\tilde{U}_{N_{k}}=\left(\tilde{v}_{N_{k}}, \tilde{\pi}_{N_{k}}, \tilde{z}, \tilde{W}\right) \in \mathcal{E}$ such that the law of each $\tilde{U}_{N_{k}}$ is $v_{N_{k}}$ and

$$
\tilde{v}_{N_{k}} \rightarrow \tilde{v} \quad \tilde{\mathbb{P}}-\text { a.s. },
$$

where $\tilde{v}$ is a random variable whose law is $\mu$.

It is easy to check that $\tilde{W}$ is a Wiener process which keeps the same regularity properties of $W$. Notice that

$$
\mathbb{P}\left[v_{N} \in L^{2}(0, T ; D(A)) \cap C([0, T] ; V)\right]=1,
$$

and so the same holds true for the new random variables $\tilde{v}_{N_{k}}$. In the same way we can deduce that $\pi_{N_{k}} \in L^{2}\left(D_{T}\right)$ and so on. Now we need to show that the $\tilde{v}_{N_{k}}$ satisfy the equations and the energy inequalities. We give a proof, using a trick of Bensoussan [1], for the local energy inequality (actually it is an equality for the $\left.v_{N}\right)$. Given $\phi \in C_{c}^{\infty}\left(D_{T}\right)$, define the random variable 


$$
\begin{aligned}
X^{N}:(\Omega, \mathcal{F}) \rightarrow & (\mathbf{R}, \mathcal{B}(\mathbf{R})) \text { as } \\
X^{N}= & \left.\left|2 \int_{0}^{T} \int_{D}\right| \nabla v_{N}\right|^{2} \varphi-\int_{0}^{T} \int_{D}\left|v_{N}\right|^{2}\left(\partial_{t} \varphi+\triangle \varphi\right) \\
& -\int_{0}^{T} \int_{D}\left(\left|v_{N}\right|^{2}+2 v_{N} \cdot z\right)\left(\left(v_{N}+z\right)^{N} \cdot \nabla \varphi\right) \\
& -2 \int_{0}^{T} \int_{D} \varphi z \cdot\left(\left(v_{N}+z\right)^{N} \cdot \nabla\right) v_{N}-\int_{0}^{T} \int_{D} 2 \pi_{N} v_{N} \cdot \nabla \varphi \mid,
\end{aligned}
$$

and let $\tilde{X}^{N_{k}}$ be the analogue of $X^{N}$ for the $\tilde{U}_{N_{k}}$.

We know that $X^{N}=0, \mathbb{P}$-a. s., and so

$$
\mathrm{E} \frac{X^{N}}{1+X^{N}}=0
$$

Notice that

$$
\frac{X^{N}}{1+X^{N}}=\Phi\left(u_{N}\right)
$$

where $\Phi$ is a deterministic bounded continuous function on the subspace of $\mathcal{E}$ where the $v_{N}$ are concentrated (remember that the $U_{N}$ are far more regular than the elements of $\mathcal{E}$ ) and so

$$
\tilde{\mathbb{E}} \frac{\tilde{X}^{N_{k}}}{1+\tilde{X}^{N_{k}}}=\tilde{\mathbb{E}} \Phi\left(\tilde{U}_{N_{k}}\right)=\int_{\mathcal{E}} \Phi(u) v_{N_{k}}(d u)=\mathbb{E} \Phi\left(U_{N_{k}}\right)=\mathbb{E} \frac{X^{N_{k}}}{1+X^{N_{k}}}=0
$$

this means

$$
\tilde{X}_{N_{k}}=0 \quad \tilde{\mathbb{P}}-\text { a.s. }
$$

If we do this for a dense set of functions in $C_{c}^{\infty}\left(D_{T}\right)$, we can conclude that there exists a set $\tilde{\Omega}_{0} \subset \tilde{\Omega}$ of full measure such that the local energy inequality holds for each $\omega \in \tilde{\Omega}_{0}$ and $\phi \in C_{c}^{\infty}\left(D_{T}\right)$.

From now on, since the two sequences enjoy the same properties, we will omit the tilde.

The last step of the proof is to show that the limit process is a martingale solution. We need to find the limit of the sequence of the pressures in such a way that the equations and the energy inequalities are satisfied. First we observe that the $v_{N_{k}}$ solve the equation

$$
\partial_{t} v_{N_{k}}+A v_{N_{k}}+B\left(\left(v_{N_{k}}\right)^{N_{k}}+z, v_{N_{k}}+z\right)=0
$$

P-a.s. in $V^{\prime}$, so in the limit

$$
\partial_{t} v+A v+B(v+z, v+z)=0, \quad \text { P-a.s in } V^{\prime} .
$$

Thus there exists a distribution $\pi$ such that (2.3) holds true. Normalise $\pi$ in such a way that

$$
\int_{D} \pi(t) d x=0 \quad \text { a.e. } t .
$$

The set of $\omega \in \Omega$ such that $W(\omega)$, and so $z(\omega)$ and all $v_{N_{k}}(\omega)$, has the suitable regularity we need, such that $\left(v_{N_{k}}(\omega), \pi_{N_{k}}(\omega)\right)$ satisfy the modified Navier-Stokes equations and such that $v_{N_{k}}(\omega) \rightarrow v(\omega)$, has probability one. Take an $\omega \in \Omega$ in this way. Then there exists a subsequence of $\pi_{N_{k}}(\omega)$ 
which converges weakly in $L_{\text {loc }}^{5 / 3}\left(D_{T}\right)$. Taking the limit in the equations, we observe that the equations are satisfied both by $\pi(\omega)$ and by the limit of $\pi_{N_{k}}(\omega)$. This means that the two are equal (they have both zero mean in $D$ ) and

$$
\pi_{N_{k}}(\omega) \rightarrow \pi(\omega) \quad \text { weakly in } L_{\text {loc }}^{5 / 3}\left(D_{T}\right)
$$

We need only to verify that $(v(\omega), \pi(\omega))$ satisfies the local energy inequality. This can be done as in the third step of the proof of Theorem 4.1, since $v_{N_{k}}(\omega)$ converges to $v(\omega)$ strongly in $L^{2}\left(D_{T}\right)$, weakly in $L^{2}\left(0, T ; H_{0}^{1}(D)\right)$ and weakly* in $L^{\infty}\left(0, T ; L^{2}(D)\right)$, while $\pi_{N_{k}}(\omega)$ converges to $\pi(\omega)$ weakly in $L_{\text {loc }}^{5 / 3}\left(D_{T}\right)$.

Finally we set $u=v+z$ and $P=\pi+Q$ and we can conclude that $(u, P)$ is a martingale suitable weak solution in the sense of Definition 2.4

4.3. The proof of Theorem 2.11. In this last section we prove the existence of stationary solutions. The proof is given using the classical KrylovBogoliubov method, where the initial measure is given by the law of a martingale solution.

In order to show the existence of time-stationary measures, we need the following compactness lemma.

Lemma 4.6. Let $\left(T_{N}\right)$ be a sequence of positive real numbers such that $T_{N} \uparrow \infty$, let $k\left(T_{N}\right)$ be an increasing sequence of positive constants and let $\beta, s, p>0$ be such that $\beta>0, s<\frac{1}{2}$ and $s p<1$. Then the set $K$ of all $(u, W) \in \mathcal{S}$ such that

$$
\|u\|_{L^{\infty}\left(0, T_{N} ; H\right)}^{2}+\|u\|_{L^{2}\left(0, T_{N} ; V\right)}^{2}+\|W\|_{W^{s, p}\left(0, T_{N} ; D\left(A^{\beta}\right)\right)}^{p} \leq k\left(T_{N}\right)
$$

for each $N \in N$ is compact in $\mathcal{S}$.

Proof. Since the $u \in K$ are bounded in $L^{\infty}(0, T ; H)$ and in $L^{2}(0, T ; V)$ and they satisfy equation (2.1) in distributions, it follows that they are bounded in $H^{1}\left(0, T ; D\left(A^{-1}\right)\right.$ (see Temam [22]).

Moreover the immersion of the space $W^{s, p}\left(0, T ; D\left(A^{\beta}\right)\right)$ in $C([0, T] ; H)$ is compact. In conclusion, for any given $T_{N}$, we need only to show that, if $\left(u_{n}, W_{n}\right) \in K$ and

$$
\left(u_{n}, W_{n}\right) \rightarrow(u, W) \quad \text { in } L^{2}\left(0, T_{N} ; H\right) \times C_{0}\left(\left[0, T_{N}\right] ; H\right)
$$

then $(u, W) \in \mathcal{S}$, that is $(u, W)$ is a suitable weak solution in $\left[0, T_{N}\right]$.

Let $z_{n}$ be the solution of the Stokes equation (2.2) with $\partial_{t} W_{n}$ as a forcing term and let $v_{n}=u_{n}-z_{n}$ and $\pi_{n}=P_{n}-Q_{n}$. By well known results on the Stokes equation (see [6]) we know that $z_{n}$ are bounded in $L^{\infty}(0, T ; H)$, $L^{2}(0, T ; V)$ and $L^{\infty}\left(0, T ; L^{4}(D)\right)$ and moreover $z_{n} \rightarrow z$ in $L^{2}(0, T ; H)$. Then $v_{n}$ are bounded in $L^{\infty}(0, T ; H) \cap L^{2}(0, T ; V)$ and so we can proceed as in the third step of the proof of Theorem 4.1 to get all the convergence properties we need to take the limit in the equations and in the local energy inequality. 
Proof of Theorem 2.11] We use the Krylov-Bogoliubov procedure for the semigroup $\tau_{t}$ in $\mathcal{S}$. Let $u_{0} \in H$. In the previous section we have shown the existence of at least one martingale suitable weak solution $\bar{u}$ of NavierStokes system driven by a Brownian motion $W$ and with initial condition $u_{0}$. Let $v_{0} \in M_{1}(S)$ be the law of the stochastic process $(\bar{u}, W)$ with values in $S$. Let $v_{t}=\tau_{t} v_{0}$ and set $\mu_{t}=\frac{1}{t} \int_{0}^{t} v_{s} d s$. Notice that the $W$-component of $v_{t}$ and $\mu_{t}$ is always the Wiener measure given by the Brownian motion $W$, due to the stationarity of this process.

Suppose that for each $\varepsilon>0$ there exists a compact set $K_{\varepsilon}$ in $\mathcal{S}$ such that

$$
\mathrm{v}_{t}\left(K_{\varepsilon}\right) \geq 1-\varepsilon \quad \text { for all } t \geq 0
$$

(this claim will be proved in the sequel of the proof), so that $\mu_{t}\left(K_{\varepsilon}\right) \geq 1-\varepsilon$, and the family of measures $\left(\mu_{t}\right)_{t \geq 0}$ is tight. By means of Prohorov theorem there is a subsequence $\left(\mu_{t_{n}}\right)_{n \in \mathbf{N}}$ which converges weakly to some $\mu \in M_{1}(S)$. The measure $\mu$ is time stationary, in fact if $t \geq 0$ and $\phi \in C_{b}(S)$,

$$
\begin{aligned}
\left(\tau_{t} \mu\right)(\phi) & =\mu\left(\tau_{t} \phi\right) \\
& =\lim _{n \rightarrow \infty} \mu_{t_{n}}\left(\tau_{t} \phi\right) \quad\left(\text { since } \tau_{t} \phi \in C_{b}(W)\right) \\
& =\lim _{n \rightarrow \infty} \frac{1}{t_{n}} \int_{0}^{t_{n}} v_{0}\left(\tau_{s+t} \phi\right) d s \\
& =\lim _{n \rightarrow \infty} \frac{1}{t_{n}} \int_{t}^{t+t_{n}} v_{0}\left(\tau_{r} \phi\right) d r \\
& =\lim _{n \rightarrow \infty} \mu_{t_{n}}(\phi)+\lim _{n \rightarrow \infty} \frac{1}{t_{n}}\left(\int_{t_{n}}^{t+t_{n}} \mathrm{v}_{0}\left(\tau_{r} \phi\right) d r-\int_{0}^{t} \mathrm{v}_{0}\left(\tau_{r} \phi\right) d r\right) \\
& =\mu(\phi) \quad\left(v_{0}\left(\tau_{r} \phi\right) \text { is bounded in } r\right) .
\end{aligned}
$$

Then we show the claim in (4.4). In order to show that $v_{t}$ is a tight family of measures, we need only to show that

$$
\mathbb{E}\left[\sup _{(0, T)}\left|\tau_{t} \bar{u}\right|_{+}^{2} \int_{0}^{T}\left\|\tau_{t} \bar{u}\right\|^{2} d t+\left\|\tau_{t} W\right\|_{W^{s, p}}^{p}\right] \leq C(T) \quad \text { uniformly in } t \geq 0
$$

in fact if we take $k\left(T_{n}\right)>2^{n+1} \varepsilon C\left(T_{n}\right)$ and $K_{\varepsilon}$ as in Lemma 4.6, we have

$$
\begin{aligned}
\mathrm{v}_{t}\left[K_{\varepsilon}^{c}\right] & =\mathbb{P}\left[\tau_{t}(\bar{u}, W) \notin K_{\varepsilon}\right] \\
& =\mathbb{P}\left[\cup_{n \in \mathbf{N}}\left\{\sup _{\left(0, T_{n}\right)}\left|\tau_{t} \bar{u}\right|_{+}^{2} \int_{0}^{T_{n}}\left\|\tau_{t} \bar{u}\right\|^{2} d t+\left\|\tau_{t} W\right\|_{W^{s, p}}^{p}>k\left(T_{n}\right)\right\}\right] \\
& \leq \sum_{n=0}^{\infty} \mathbb{P}\left[\sup _{\left(0, T_{n}\right)}\left|\tau_{t} \bar{u}\right|_{+}^{2} \int_{0}^{T_{n}}\left\|\tau_{t} \bar{u}\right\|^{2} d t+\left\|\tau_{t} W\right\|_{W^{s, p}}^{p}>k\left(T_{n}\right)\right] \\
& \leq \sum_{n=0}^{\infty} \frac{1}{k\left(T_{n}\right)} \mathbb{E}\left[\sup _{\left(0, T_{n}\right)}\left|\tau_{t} \bar{u}\right|_{+}^{2} \int_{0}^{T_{n}}\left\|\tau_{t} \bar{u}\right\|^{2} d t+\left\|\tau_{t} W\right\|_{W^{s, p}}^{p}\right] \\
& \leq \sum_{n=0}^{\infty} \frac{C\left(T_{n}\right)}{k\left(T_{n}\right)}<\varepsilon .
\end{aligned}
$$


To prove the claim, we use (2.5) and Poincaré inequality to get

$$
\mathbb{E}|\bar{u}(t)|_{H}^{2}+\lambda \mathbb{E} \int_{s}^{t}|\bar{u}(r)|_{H}^{2} d r \leq \mathbb{E}|\bar{u}(s)|_{H}^{2}+\left(\sigma+\|f\|_{V^{\prime}}^{2}\right)(t-s),
$$

then using Lemma 4.9 it follows that

$$
\mathbb{E}|\bar{u}(t)|^{2} \leq \sup _{s \in(0,1)} \mathbb{E}|\bar{u}(s)|^{2}+\frac{\sigma+\|f\|_{v^{\prime}}}{\lambda^{2}} \quad \text { for almost every } t .
$$

Finally by (2.6) we obtain

$$
\begin{aligned}
\mathbb{E}\left|\tau_{t} \bar{u}\right|_{L^{\infty}(0, T ; H) \cap L^{2}(0, T ; V)}^{2}= & \mathbb{E}\left[\sup _{(t, t+T)}|\bar{u}(s)|_{H}^{2}+\int_{t}^{t+T}\|\bar{u}(s)\|_{V}^{2} d s\right] \\
\leq & 2 \mathbb{E}|\bar{u}(t)|_{H}^{2}+2\left(\|f\|_{V^{\prime}}^{2}+\sigma\left(1+\sigma C_{1}^{2}\right)\right) T \\
\leq & 2 \sup _{s \in(0,1)} \mathbb{E}|\bar{u}(s)|^{2}+\frac{2 \sigma+\|f\|_{V^{\prime}}}{\lambda^{2}} \\
& +2\left(\|f\|_{V^{\prime}}^{2}+\sigma\left(1+\sigma C_{1}^{2}\right)\right) T .
\end{aligned}
$$

The estimate on the Brownian motion is classical:

$$
\begin{aligned}
\mathbb{E}\left\|\tau_{t} W\right\|_{W^{s, p}}^{p} \leq & \int_{0}^{T} \mathbb{E}\left\|\tau_{t} W(r)\right\|_{D\left(A^{\beta}\right)}^{p} d r \\
& +\int_{0}^{T} \int_{0}^{T} \frac{\mathbb{E}\left\|\tau_{t} W\left(r_{1}\right)-\tau_{t} W\left(r_{2}\right)\right\|_{D\left(A^{\beta}\right)}^{p}}{\left|r_{1}-r_{2}\right|^{1+s p}} d r_{1} d r_{2} \\
\leq & C_{p} \int_{0}^{T} r^{p / 2} d r+C_{p} \int_{0}^{T} \int_{0}^{T}\left|r_{1}-r_{2}\right|^{\left(\frac{1}{2}-s\right) p-1} d r_{1} d r_{2} \\
\leq & C_{p, T},
\end{aligned}
$$

since $s<\frac{1}{2}$.

We want to show now that the stationary measure has finite mean dissipation rate. We consider

$$
\phi(u)=\int_{0}^{T}\|u(t)\|^{2} d t
$$

this is a lower semi-continuous function on $\mathcal{S}$, then there exists an increasing sequence of functions $\phi_{N} \in C_{b}(S)$ such that $\phi_{N} \uparrow \phi$. From the monotone convergence theorem $\left\langle\phi_{N}, \mu\right\rangle$ converges to $\langle\phi, \mu\rangle$, even if the last term is not finite. So it is sufficient to show that $\left\langle\phi_{N}, \mu\right\rangle$ is bounded independently from $N$. Now

and by (4.5),

$$
\left\langle\phi_{N}, \mu\right\rangle=\lim _{n \rightarrow \infty} \frac{1}{t_{n}} \int_{0}^{t_{n}}\left\langle\phi_{N}, v_{s}\right\rangle d s
$$

$$
\left\langle\phi_{N}, v_{s}\right\rangle=\mathbb{E} \phi_{n}\left(\tau_{s}(\bar{u})\right) \leq \mathbb{E} \phi\left(\tau_{s}(\bar{u})\right) \leq \mathbb{E}\left\|\tau_{s}(\bar{u})\right\|_{L^{2}(0, T ; V)} \leq C_{T} .
$$

Finally we show (2.7). Let

$$
\Theta(t)=\int_{\mathcal{S}}\left[\int_{0}^{t}\|u\|^{2}\right] \mu(d u)
$$


then by the invariance of $\mu$,

$$
\begin{aligned}
\Theta(t)-\Theta(s) & =\int_{\mathcal{S}} \int_{0}^{t-s}\|u(r+s)\|^{2} d r \mu(d u) \\
& =\int_{\mathcal{S}} \int_{0}^{t-s}\|u(r)\|^{2} d r \mu(d u) \\
& =\Theta(t-s) .
\end{aligned}
$$

Since $\Theta$ is non decreasing, then $\Theta(t)=C t$.

Remark 4.7. When the dynamic is well defined, one can be interested in studying other mathematical objects, which can give some asymptotic information on the solutions. For example the dynamic for the linear Stokes equations is well defined and one can study the invariant measures of this equation. Then it can be easily seen that any time-stationary solution in the path space, frozen at an arbitrary time, is an invariant measure. In fact let

$$
p_{T}: z \mapsto z(T): C([0, \infty) ; H) \rightarrow H,
$$

such mapping is continuous. Let $\mu^{z}$ be the the time-invariant measure which can be built for the Stokes equation.

Proposition 4.8. The image measure of $\mu^{z}$ through $p_{T}$ is an invariant measure for the Stokes equation (2.2).

Proof. By the proof of the previous theorem

$$
\mu^{z}=\lim _{n \rightarrow \infty} \frac{1}{t_{n}} \int_{0}^{t_{n}} \tau_{s} v_{0}^{z} d s
$$

where $v_{0}^{z}$ is the law of $\omega \rightarrow z(\cdot, \omega)$ in $C([0, \infty] ; H)$. Then

$$
p_{T} \mu^{z}=\lim _{n \rightarrow \infty} \frac{1}{t_{n}} \int_{0}^{t_{n}} p_{T} \tau_{s} v_{0}^{z} d s=\lim _{n \rightarrow \infty} \frac{1}{t_{n}} \int_{0}^{t_{n}} p_{T+s} v_{0}^{z} d s,
$$

since $p_{T} \circ \tau_{s}=p_{T+s}$. Now, since $v_{0}^{z}$ is the law of $z(\cdot, 0)$, then $p_{T+s} v_{0}^{z}$ is the law of $z(T+s, 0)$, that is the law of $z(s, z(T, 0))$. In conclusion

$$
p_{T} \mu^{z}=\lim _{n \rightarrow \infty} \frac{1}{t_{n}} \int_{0}^{t_{n}} \mathcal{L} z(s, z(T, 0)) d s
$$

and, by Proposition 11.3 of [5], $p_{T} \mu^{z}$ is an invariant measure.

Finally we prove the easy exotic Gronwall lemma we used in the proof of the previous theorem.

Lemma 4.9. Suppose the function $v:[0,+\infty) \rightarrow \mathbf{R}$ satisfies

$$
v(t) \leq v(s)-\lambda \int_{s}^{t} v(r) d r+C(t-s)
$$

for all $t \geq 0$ and almost all $s \leq t$. Then

$$
v(t) \leq \sup _{s \in(0,1)} v(s)+\frac{C}{\lambda}
$$

for almost all $t \geq 0$. 
Proof. Let $t>0$ and let $\mathcal{N}$ be the set of Lebesgue measure zero for which the inequality does not hold. Set $u(s)=-v(t-s)$ for $s \in[0, t]$. It is easy to see that for each $s \in[0, t]$ such that $t-s \notin \mathcal{N}$, we have

$$
u(s) \leq u(0)+\lambda \int_{0}^{s} u(r) d r+C s
$$

and, by Gronwall lemma

$$
u(s) \leq u(0) \mathrm{e}^{\lambda s}+\frac{C}{\lambda}\left(\mathrm{e}^{\lambda s}-1\right)
$$

This means

$$
v(t) \leq v(t-s) \mathrm{e}^{-\lambda s}+\frac{C}{\lambda}\left(1-\mathrm{e}^{-\lambda s}\right) \leq v(t-s)+\frac{C}{\lambda},
$$

and then we can conclude that

$$
v(t) \leq \sup _{s \in(0,1)} v(s)+\frac{C}{\lambda} .
$$

\section{REFERENCES}

1. A. Bensoussan, Stochastic Navier-Stokes equations, Acta Appl. Math. 38 (1995), 267-304.

2. H. BEIRAO DA VEIGA, On the construction of suitable weak solutions to the Navier Stokes equations via a general approximation theorem, J. Math. Pures Appl., IX. Ser. 64 (1985), 321-334.

3. N. Bourbaki, Topologie générale, Éléments de Mathématique, Hermann, Paris (1958).

4. L. Caffarelli, R. Kohn, L. Nirenberg, Partial regularity of suitable weak solutions of the Navier-Stokes equations, Comm. Pure Appl. Math. XXXV (1982), 771831.

5. G. DA Prato, J. ZAbCZYK, Stochastic equations in infinite dimension, Cambridge Univ. Press, Cambridge 1992.

6. F. Flandoli, Stochastic differential equations in fluid dynamics, Rendiconti del Seminario Fisico-Matematico di Milano (1996).

7. F. Flandoli, M. Romito, Statistically stationary solutions to the 3D Navier-Stokes equations do not show singularities, Elec. J. Prob. 6 (2001).

8. F. Flandoli, M. Romito, Partial regularity for the stochastic Navier-Stokes equations, Trans. Amer. Math. Soc. 354, no. 6 (2002), 2207-2241.

9. F. Flandoli, B. Schmalfuss, Weak solutions and attractors for the $3 D$ NavierStokes equations with non-regular force, J. Dynam. Diff. Eq. 11, Nr. 2 (1999), 355398.

10. N. IKedA, S. WATANABE, Stochastic differential equations and diffusion processes, North Holland Mathematical Library 24, North Holland/Kodansha (1989).

11. P. G. LEMARIE-RIEUSSET, Solutions faibles d'energie infinie pour les equations de Navier-Stokes dans $\mathbf{R}^{3}$, C. R. Acad. Sci., Paris Ser. I, Math. 328, No. 12 (1999), 11331138 .

12. F. Lin, A new proof of the Caffarelli-Kohn-Nirenberg theorem, Comm. Pure Appl. Math. LI (1998), 241-257.

13. P. L. LiOnS, Mathematical topics in fluid dynamic, Vol. 1, Clarendon Press, Oxford (1996). 
14. E. PARdoux, Equations aux derivees partielles stochastiques nonlineaires monotones. Etude de solutions fortes de type Ito, These, Université Paris Sud, Novembre 1975.

15. M. Romito, Partial regularity theory for a stochastic Navier-Stokes system, Thesis, Pisa (2000).

16. V. SCHEFFER, Partial regularity of solutions to the Navier-Stokes equations, Pacific J. Math. 66 (1976), 532-552.

17. V. SCHEFFER, Hausdorff measure and the Navier-Stokes equations, Comm. Math. Phys. 61 (1978), 41-68.

18. V. SCHEFFER, The Navier-Stokes equation on a bounded domain, Comm. Math. Phys. 73 (1980), 1-42.

19. G. SELL, Global attractor for the 3D Navier-Stokes equations, J. Dynam. Diff. Eq. 8 (1), 1996.

20. V. A. SolonNikov, Estimates of the solutions of a nonstationary linearized system of Navier-Stokes equations, Amer. Math. Soc. Translations, Ser. 2, Vol. 75, 1-117.

21. H. SOHR, W. VON WAHL, On the regularity of the pressure of weak solutions of Navier-Stokes equations, Arch. Math. 46, Basel (1986), 28-439.

22. R. Temam, The Navier-Stokes Equations, North Holland, 1977.

23. R. Temam, Navier-Stokes Equations and Nonlinear Functional Analysis, SIAM, Philadelphia, 1983.

24. R. Temam, Infinite Dimensional Dynamical Systems in Mechanics and Physics, Springer-Verlag, New York, 1988.

Dipartimento di Matematica U. Dini, Università di Firenze, Viale MorGAGNI 67/A, 50134 FIRENZE, ITALIA

E-mail address: romito@math.unifi.it 